\title{
Atomistic Long-Term Simulation of Heat and Mass Transport
}

\author{
G. Venturini ${ }^{\mathrm{a}}$, K. Wang ${ }^{\mathrm{b}}$, I. Romero ${ }^{\mathrm{c}}$, M. P. Ariza ${ }^{\mathrm{d}}$, M. Ortiz $^{\mathrm{a}, *}$ \\ ${ }^{a}$ Division of Engineering and Applied Science, California Institute of Technology \\ Pasadena, CA 91125, USA \\ ${ }^{b}$ Department of Aerospace and Ocean Engineering, Virginia Polytechnic Institute and \\ State University Blacksburg, VA 24061, USA \\ ${ }^{c}$ Escuela Técnica Superior de Ingenieros Industriales, Universidad Politécnica de \\ Madrid, 28006 Madrid, Spain \\ ${ }^{d}$ Escuela Técnica Superior de Ingeniería, Universidad de Sevilla, 41092 Sevilla, Spain
}

\begin{abstract}
We formulate a theory of non-equilibrium statistical thermodynamics for ensembles of atoms or molecules. The theory is an application of Jayne's maximum entropy principle, which allows the statistical treatment of systems away from equilibrium. In particular, neither temperature nor atomic fractions are required to be uniform but instead are allowed to take different values from particle to particle. In addition, following the Coleman-Noll method of continuum thermodynamics we derive a dissipation inequality expressed in terms of discrete thermodynamic fluxes and forces. This discrete dissipation inequality effectively sets the structure for discrete kinetic potentials that couple the microscopic field rates to the corresponding driving forces, thus resulting in a closed set of equations governing the evolution of the system. We complement the general theory with a variational meanfield theory that provides a basis for the formulation of computationally tractable approximations. We present several validation cases, concerned with equilibrium properties of alloys, heat conduction in silicon nanowires and hydrogen desorption from palladium thin films, that demonstrate the range and scope of the method and assess its fidelity and predictiveness. These validation cases are characterized by the need or desirability to account for atomiclevel properties while simultaneously entailing time scales much longer than
\end{abstract}

${ }^{*}$ Corresponding author

E-mail address: ortiz@caltech.edu (M. Ortiz).

Preprint submitted to Journal of the Mechanics and Physics of Solids

May 28, 2014

(C) 2014. This manuscript version is made available under the Elsevier user license http://www.elsevier.com/open-access/userlicense/1.0/ 
those accessible to direct molecular dynamics. The ability of simple meanfield models and discrete kinetic laws to reproduce equilibrium properties and long-term behavior of complex systems is remarkable.

Keywords:

Non-equilibrium statistical thermodynamics, Binary alloys, Meanfield

theory, Variational updates, Slow kinetic processes

\section{Introduction}

In a number of areas of application, the behavior of systems depends sensitively on properties that pertain to the atomistic scale, i. e., the angstrom and femtosecond scales. Specifically, atomistic details are important where material response is highly non-linear, e. g., at atomistically-sharp crack tips and other stress concentrators, near atomic-scale defects such as vacancies and dislocations and at other atomistically-sharp features such as free surfaces, grain boundaries and material interfaces (Gill, 2010). However, often the properties and behaviors of interest are macroscopic and take place on the scale of centimeters to meters, and are characterized by slow evolution on the scale of minutes to years. A case in point is degradation in nuclear reactor materials, which results from a combination of heat, irradiation, stress and corrosion exposure for extended periods of time (20 or more years, cf., e. g., Marquis et al. (2009) and references therein). This vast disparity of length and time scales poses extraordinary challenges in theoretical and computational material science.

Molecular Dynamics (MD) and Monte Carlo (MC) methods are powerful techniques to study deformation and diffusion mechanisms in systems of particles, but they are limited to relatively small material samples and to time windows of microseconds at best (e. g., Sutton et al. (1992); Wang et al. (1992)). Considerable effort has been devoted to accelerating MD and MC methods and notable successes have been recorded in that direction (cf., e. g., Voter et al. (2002)). However, no computationally-tractable atomisticallybased models appear to be as yet available to study slow phenomena, over time scales of the order of minutes to years, while maintaining a strictly atomistic description of the material.

The present work is concerned primarily with the development of a theory of non-equilibrium statistical thermodynamics for ensembles of atoms or molecules that addresses that theoretical and computational gap. The theory 
provides a framework for the development of computational models capable of simulating slow thermo-chemo-mechanical processes, such as corrosion or segregation of impurities, that occur over time-windows that are intractable by means of the existing atomistic or atomistically-informed models. In particular, the theory effectively accounts for thermal and stoichiometric effects, including heat and mass transport, without the need to track thermal vibrations and individual particle hops explicitly. Given the advantageous properties of numerical methods based on variational formulations, special emphasis is placed in obtaining variational principles that characterize the state or evolution in the system.

The proposed theory is an application of Jayne's maximum entropy principle (Jaynes, 1957a,b) that allows the statistical treatment of systems away from equilibrium. By maximizing a suitable constrained entropy, effective thermodynamic potentials are identified which characterize the instantaneous state of a system and supply driving forces for its evolution. Specifically, we formulate a grand-canonical free entropy that, unlike other conventional thermodynamic potentials such as the free energy, is well-defined away from equilibrium. In addition, the stationarity of the grand-canonical free entropy characterizes the states of thermodynamic equilibrium of the system. In practice, the calculation of the grand-canonical partition function in closed form, when all the local equilibrium relations derive, is generally intractable, which raises the need for approximation theory. Variational meanfield theory is often used to devise computationally tractable approximations to complex thermodynamical systems (Callen, 1985; Yeomans, 1992). By exploiting the optimality properties of the theory, meanfield models can systematically be developed and Bogoliubov-like variational principles can be proven, even away from equilibrium. In particular, we show that trial meanfield Hamiltonians always lead to guaranteed lower bounds of the grand-canonical free entropy, and that this thermodynamic potential is well defined both in equilibrium and away from it.

The fundamental difference between the present theory and previous approaches based on free-energy minimization (cf., e. g. Sutton et al. (1992); Wang et al. (1992)) is that neither temperature nor atomic fractions are required to be uniform but instead are allowed to take different values from particle to particle. Following Kulkarni et al. (2008), we additionally characterize the evolution of the system, be it towards equilibrium or in response to external driving, by means of kinetic equations of the Onsager type (Onsager, 1931a,b; De Groot and Mazur, 1962). We develop this discrete kinetic 
framework by mirroring the classical Coleman-Noll method of continuum thermodynamics (Coleman and Noll, 1963), with the aim of deriving a dissipation inequality expressed in terms of discrete thermodynamic fluxes and forces. This discrete dissipation inequality effectively identifies the appropriate form of said thermodynamic fluxes and forces and their duality pairings. These pairings in turn set the structure of discrete kinetic potentials that couple the microscopic field rates to the corresponding driving forces, thus resulting in a closed set of equations governing the evolution of the system.

Within the framework just outlined, the discrete kinetic potentials are empirical and subject to modeling, much in the same way as the interatomic potentials used in practice are also empirical and the result of modeling. For instance, Kulkarni et al. (2008) proposed a class of linear discrete kinetic equations based on interpolation of the atomistic fields and subsequent use of continuum transport equations such as Fourier's law of heat conduction. The advantage of such discrete models is that they can be calibrated directly against experimentally measured macroscopic transport properties, such as thermal conductivity, while simultaneously affording a measure of atomistic realism. In this work, we develop a general framework for discrete linear kinetics encompassing both heat and mass transport. A general connection between atomistic and macroscopic transport coefficients, such as thermal conductivities and diffusivities, can be forged by means of a standard asymptotic analysis of the long-wavelength limit of the discrete governing equations (cf., e. g., Askar (1986)). For some simple geometries and sufficiently short-range interactions, this discrete-to-continuum long-wavelength limiting relations suffice to determine all the atomic-level transport constants, thus allowing for a direct experimental calibration of the models.

In addition to formulating the theoretical framework and attendant meanfield approximation theory, we present several validation cases aimed at demonstrating the range and scope of the method and assessing its fidelity and predictiveness. A first concern in this regard is how well simple meanfield models, such as must necessarily be used in practice, approximate thermodynamic driving forces and, in particular, equilibrium properties. We ascertain this question by formulating a simple meanfield model for substitutional alloys and assessing its ability to reproduce equilibrium properties of four alloys, including lattice parameter, linear thermal expansion coefficient, elastic moduli and surface segregation concentration. A second concern is how well empirical discrete kinetic equations can model long-term process in complex systems. Again, we address this question by means of two validation 
cases: Heat conduction in silicon nanowires and hydrogen storage in palladium. The first case tests the ability of the theory to model heat conduction and the second mass transport in applications requiring atomistic resolution while, simultaneously, involving time scales much longer than typically accessible to molecular dynamics. Evidently, heat conduction at the nanoscale and hydrogen storage in metals are vast areas of research and technological development in their own right. The narrow aim of the present study is not to advance those areas of endeavor but to borrow from them representative validation cases. In the same spirit, a general numerical implementation of the theory, possibly including spatial coarse-graining, is beyond the scope of this work. Instead, we rely on simple models that are analytically tractable for the strict purposes of validation.

The organization of the paper is as follows. Section 2 presents a max-ent theory of non-equilibrium statistical mechanics and thermodynamics of particulate systems containing multiple species. Section 3 formulates the kinetic relations that drive the evolution of the thermodynamic field variables. A variational meanfield approximation framework is presented in Section 4 that effectively extends classical variational meanfield theory away from equilibrium. Validation examples focusing on equilibrium properties of alloys are presented in Section 5. Validation examples focusing on long-term kinetics, including heat conduction and mass transport, are presented in Section 6. A summary of the theory and a discussion of its strengths and limitations and of possible extensions and further work is finally presented in Section 7 .

\section{Maximum entropy and local equilibrium relations}

We begin by formulating an extension of the classical maximum-entropy formalism of equilibrium statistical mechanics (Jaynes, 1957a,b) that encompasses systems away from equilibrium. We accomplish this extension by recourse to Jaynes' principle of maximum entropy and to variational meanfield theory (cf., e. g., Yeomans (1992) for a concise account of the classical theory). The aim of the non-equilibrium extension is to account for thermal oscillations and mass transport without solving the equations of motion or tracking individual jumps of the particles. In particular, we wish to identify the consistent thermodynamic forces that drive kinetics and the evolution of the system.

We specifically consider a system consisting of $N$ particles, e. g., atoms or molecules, each of which can be of one of $M$ species. For each particle 
$i=1, \ldots, N$, and each species $k=1, \ldots, M$, we introduce the occupancy function

$$
n_{i k}= \begin{cases}1, & \text { if site } i \text { is occupied by species } k, \\ 0, & \text { otherwise, }\end{cases}
$$

in order to describe the occupancy of each site. We note that, from definition (2.1), we must have

$$
\sum_{k=1}^{M} n_{i k}=1
$$

at every site $i$. We shall additionally denote by $\boldsymbol{n}_{i}=\left(n_{i k}\right)_{k=1}^{M}$ the local occupancy array of site $i$. Thus, the microscopic states of the system are defined by the instantaneous position $\{\boldsymbol{q}\}=\left(\boldsymbol{q}_{i}\right)_{i=1}^{N}$, momenta $\{\boldsymbol{p}\}=\left(\boldsymbol{p}_{i}\right)_{i=1}^{N}$, and occupancy arrays $\{\boldsymbol{n}\}=\left(\boldsymbol{n}_{i}\right)_{i=1}^{N}$ of all $N$ particles in the system. It follows from definition (2.1) that the occupancy functions $\boldsymbol{n}_{i}$ take values in a set, denoted $\mathcal{O}_{M}$, consisting of the elements of $\{0,1\}^{M}$ that satisfy the constraint (2.2). In addition, the occupancy arrays $\{\boldsymbol{n}\}$ take values in the set

$$
\mathcal{O}_{N M}=\left\{\{\boldsymbol{n}\} \in\{0,1\}^{N M}, \boldsymbol{n}_{i} \in \mathcal{O}_{M} \text { for } i=1, \ldots, N\right\} .
$$

Now suppose that a function $A(\{\boldsymbol{q}\},\{\boldsymbol{p}\},\{\boldsymbol{n}\})$ represents a physical property of the system. The expected or macroscopic value of $A$ is given by the phase average

$$
\langle A\rangle=\sum_{\{\boldsymbol{n}\} \in \mathcal{O}_{N M}} \frac{1}{h^{3 N}} \int_{\Gamma} A(\{\boldsymbol{q}\},\{\boldsymbol{p}\},\{\boldsymbol{n}\}) \rho(\{\boldsymbol{q}\},\{\boldsymbol{p}\},\{\boldsymbol{n}\}) d q d p
$$

where $\Gamma=\left(\mathbb{R}^{3} \times \mathbb{R}^{3}\right)^{N}, h$ is Planck's constant and $h^{-3 N}$ supplies the natural unit of phase volume for systems of distinguishable particles. ${ }^{1}$ In (2.4), the function $\rho(\{\boldsymbol{q}\},\{\boldsymbol{p}\},\{\boldsymbol{n}\})$ is a probability density that satisfies the normalization condition

$$
\langle\rho\rangle=1
$$

and we write

$$
d q d p=\prod_{i=1}^{N} \prod_{j=1}^{3} d q_{i j} d p_{i j}
$$

\footnotetext{
${ }^{1}$ cf., e. g., Hill (1987); Girifalco (2000) for discussions on particle distinguishability and its role in statistical mechanics.
} 
for the element of phase space volume (Hill, 1962; Girifalco, 2000).

In this work, we assume that the statistics of the system obeys Jaynes' principle of maximum entropy (Jaynes (1957a,b), see also Zubarev (1974); Callen (1985)). This principle postulates that the probability density function $\rho(\{\boldsymbol{q}\},\{\boldsymbol{p}\},\{\boldsymbol{n}\})$, characterizing the probability of finding the system in a state $(\{\boldsymbol{q}\},\{\boldsymbol{p}\},\{\boldsymbol{n}\})$, maximizes the information-theoretical entropy

$$
\mathcal{S}[\rho]=-k_{B}\langle\log \rho\rangle,
$$

among all probability measures consistent with the constraints on the system. In (2.7) and subsequently, $k_{B}$ denotes Bolzmann's constant. It is known from information theory that the maximum-entropy, or max-ent for short, probability density function is indeed the least biased probability density function consistent with the constraints on the system (Jaynes, 1957a,b).

We specifically consider systems consisting of distinguishable particles whose Hamiltonians have the additive structure ${ }^{2}$

$$
H=\sum_{i=1}^{N} h_{i},
$$

where $h_{i}$ is the local Hamiltonian of particle $i$. Note, however, that each local hamiltonian $h_{i}$ is allowed to depend on the positions, momenta, and species of all the particles in the system. Suppose that the expected particle energies,

$$
\left\langle h_{i}\right\rangle=e_{i}
$$

and the expected particle atomic fractions

$$
\left\langle n_{i k}\right\rangle=x_{i k},
$$

$i=1, \ldots, N, k=1, \ldots, M$, are known. We note that, since the occupancies belong to the set $\mathcal{O}_{N M}$, the local atomic fractions must necessarily satisfy the identities

$$
\sum_{k=1}^{M} x_{i k}=1, \quad i=1, \ldots, N .
$$

\footnotetext{
${ }^{2}$ Other additive decompositions of the Hamiltonian, e. g., bondwise, clusterwise and others, can be treated mutatis mutandis. The particle-wise decomposition of the present work has the distinguishing property that it results in particle-wise fields and is adopted for definiteness.
} 
It bears emphasis that, unlike the classical equilibrium framework where only global constraints are enforced, the preceding constraints are local.

Enforcing the constraints (2.9) and (2.10) by means of Lagrange multipliers $k_{B}\{\beta\} \equiv\left(k_{B} \beta_{i}\right)_{i=1}^{N}$ and $k_{B}\{\gamma\} \equiv\left(k_{B} \gamma_{i}\right)_{i=1}^{N}$, with $\gamma_{i}=\left(\gamma_{i k}\right)_{k=1}^{M}$, respectively, leads to the Lagrangian

$$
\mathcal{L}[\rho,\{\beta\},\{\boldsymbol{\gamma}\}]=\mathcal{S}[\rho]-k_{B}\{\beta\}^{T}\{\langle h\rangle\}+k_{B}\{\boldsymbol{\gamma}\}^{T}\{\langle\boldsymbol{n}\rangle\}
$$

where we write $\{\langle h\rangle\} \equiv\left(\left\langle h_{i}\right\rangle\right)_{i=1}^{N}$ and $\{\langle\boldsymbol{n}\rangle\} \equiv\left(\left\langle\boldsymbol{n}_{i}\right\rangle\right)_{i=1}^{N}$. In view of identities (2.11), we append the restrictions

$$
\sum_{k=1}^{M} \gamma_{i k}=0
$$

in order to render $\{\boldsymbol{\gamma}\}$ determinate. Maximizing $\mathcal{L}[\cdot,\{\beta\},\{\gamma\}]$ among probability measures results, after a trite calculation, in

$$
\rho=\frac{1}{\Xi} \mathrm{e}^{-\{\beta\}^{T}\{h\}+\{\boldsymbol{\gamma}\}^{T}\{\boldsymbol{n}\}},
$$

where

$$
\Xi=\sum_{\{\boldsymbol{n}\} \in \mathcal{O}_{N M}} \frac{1}{h^{3 N}} \int_{\Gamma} \mathrm{e}^{-\{\beta\}^{T}\{h\}+\{\boldsymbol{\gamma}\}^{T}\{\boldsymbol{n}\}} d q d p .
$$

By analogy to equilibrium statistical mechanics, we may interpret (2.14) and (2.15) as non-equilibrium generalizations of the Gibbs grand-canonical probability density function and the grand-canonical partition function, respectively. As in the equilibrium case, the solution (2.14) of the max-ent problem is unique due to the concavity of the entropy and the linearity of the constraints (cf., e. g., Rockafellar (1970)). The value of the Lagrange multipliers $\{\beta\}$ and $\{\gamma\}$ can be determined in terms of the energy field $\{e\}$ and the atomic-fraction field $\{\boldsymbol{x}\}$ by enforcing constraints (2.9) and (2.10). Alternatively, we may regard the Lagrange multipliers $\{\beta\}$ and $\{\gamma\}$ as free parameters, whereupon the corresponding energy field $\{e\}$ and atomic-fraction field $\{\boldsymbol{x}\}$ then follow from relations (2.9) and (2.10). Also by analogy with equilibrium statistical mechanics, we may interpret

$$
\theta_{i}=\frac{1}{k_{B} \beta_{i}}
$$


as the particle absolute temperature and

$$
\boldsymbol{\mu}_{i}=\frac{\gamma_{i}}{\beta_{i}}=k_{B} \theta_{i} \gamma_{i}
$$

as the particle chemical-potential array of particle $i$, respectively. However, unlike equilibrium statistical mechanics, the temperature and chemicalpotential fields need not be uniform and may vary from particle to particle when the system is away from equilibrium. In addition, the total physical entropy $S$ may be identified with the information entropy at the grandcanonical distribution, namely,

$$
S=k_{B}\{\beta\}^{T}\{e\}-k_{B}\{\boldsymbol{\gamma}\}^{T}\{\boldsymbol{x}\}+k_{B} \log \Xi .
$$

As in the equilibrium case, a straightforward calculation gives the local or particle equilibrium relations

$$
\begin{aligned}
\beta_{i} & =\frac{1}{k_{B}} \frac{\partial S}{\partial e_{i}}(\{e\},\{\boldsymbol{x}\}), \\
\gamma_{i k} & =-\frac{1}{k_{B}} \frac{\partial S}{\partial x_{i k}}(\{e\},\{\boldsymbol{x}\}) .
\end{aligned}
$$

which relate the particle energies and atomic fractions to the particle temperatures and chemical potentials. Evidently, relations (2.18) and (2.19) generalize the classical equilibrium relations between temperature and internal energy and between chemical potentials and numbers of particles, respectively.

In the expression (2.18) of the entropy, the independent variables are the particle energies and atomic fractions. As in the equilibrium theory, it is sometimes convenient to work with the grand-canonical free entropy $\Phi$, which can be obtained either by performing a Legendre transform of the entropy (2.18) with respect to the particle energies and atomic fractions, or, directly, as

$$
\Phi(\{\beta\},\{\gamma\})=\sup _{\rho} \mathcal{L}[\rho,\{\beta\},\{\gamma\}]=k_{B} \log \Xi(\{\beta\},\{\gamma\}),
$$

with the properties

$$
\begin{aligned}
e_{i} & =-\frac{1}{k_{B}} \frac{\partial \Phi}{\partial \beta_{i}}(\{\beta\},\{\gamma\}), \\
x_{i k} & =\frac{1}{k_{B}} \frac{\partial \Phi}{\partial \gamma_{i k}}(\{\beta\},\{\gamma\}) .
\end{aligned}
$$


Again, relations (2.20) and (2.21) generalize their classical equilibrium counterparts.

\section{Detailed balance and kinetic relations}

Evidently, the grand-canonical distribution of classical equilibrium statistical mechanics is recovered from (2.14) when $\beta_{i}=\beta=$ constant and $\gamma_{i k}=\gamma_{k}=$ constant, $i=1, \ldots, N, k=1, \ldots, M$, i. e., when the temperature and chemical-potential fields are uniform, or in thermodynamic equilibrium. Since, for large systems no external agency can effect the type of local energy and atomic fraction control implied by the constraints (2.9) and (2.10), we may expect such systems to exhibit a general trend towards thermodynamic equilibrium. However, this trend towards equilibrium may also be expected to be controlled by kinetics and, therefore, require the passage of time. In particular, the behavior of systems away from equilibrium may be expected to be rheological or time dependent. For the systems of particles under consideration here the dominant kinetics may be expected to take the form of heat conduction and mass transport.

A long-standing challenge in theoretical physics is to describe such kinetic processes from first principles, be they classical molecular dynamics, or time-dependent quantum mechanical descriptions of electronic structure (cf., e. g., Ziman (1960) for a classical treatise). Such aims are beyond the scope of this work. Instead, we proceed to describe kinetics empirically within the framework of Onsager theory (Onsager, 1931a,b). Thus, in analogy with the Coleman-Noll method of continuum thermodynamics (Coleman and Noll, 1963), we aim to formulate a dissipation inequality expressed in terms of thermodynamic fluxes and forces. The duality pairings established by the dissipation inequality then provide the foundation for the formulation of discrete kinetic equations, specifically, discrete forms of Fourier's law of heat conduction and Fick's law of mass transport. These kinetic equations, when inserted into local energy-balance and mass-balance equations, define an evolution of the system. In subsequent derivations, we follow and adapt the notation of Weiner (2002).

\subsection{Local balance and local dissipation inequality}

We begin by examining the balance of energy at the particle level. The internal energy of particle $i$ may be identified with the expected value of the 
corresponding particle Hamiltonian, i. e.,

$$
u_{i}=\left\langle h_{i}\right\rangle \text {, }
$$

$i=1, \ldots, N$. Suppose, in addition, that the local Hamiltonians $h_{i}$ depend on macroscopic variables $\{\mathcal{A}\}=\left(\mathcal{A}_{1}, \ldots, \mathcal{A}_{\nu}\right)$, such as volume or deformation. Then, the balance of energy at particle $i$ can be expressed as

$$
\dot{u}_{i}=\dot{w}_{i}+\boldsymbol{\mu}_{i}^{T} \dot{\boldsymbol{x}}_{i}+r_{i}
$$

where

$$
\dot{w}_{i}=\sum_{\alpha=1}^{\nu}\left\langle\frac{\partial h_{i}}{\partial \mathcal{A}_{\alpha}} \dot{\mathcal{A}}_{\alpha}\right\rangle
$$

is the external mechanical power and $r_{i}$ is the heat flow into particle $i$.

We shall assume that the heat flow $r_{i}$ into particle $i$ is of the form

$$
r_{i}=\sum_{j \neq i} R_{i j}
$$

where the sum extends to all particles different from $i$, and

$$
R_{i j}=-R_{j i}
$$

is the discrete heat flux from particle $j$ to $i$. Likewise, we shall assume that the mass flow into particle $i$ may be expressed as

$$
\dot{\boldsymbol{x}}_{i}=\sum_{j \neq i} \boldsymbol{J}_{i j}
$$

where

$$
\boldsymbol{J}_{i j}=-\boldsymbol{J}_{j i}
$$

is the discrete mass flux array from particle $j$ to $i$.

Next, we seek to formulate a balance of entropy and a dissipation inequality at the particle level. To this end, we regard each particle as a system in equilibrium with its surroundings, regarded as a heat bath and particle repository, and we expect dissipation to arise as a result of heat and mass flux between particles. In analogy with continuum thermodynamics (cf., e. g., Malvern (1969)), we may then define a particle entropy rate $\dot{s}_{i}$ by means of the relation

$$
\frac{\dot{s}_{i}}{k_{B} \beta_{i}}=\dot{u}_{i}-\dot{w}_{i}-\boldsymbol{\mu}_{i}^{T} \dot{\boldsymbol{x}}_{i}
$$


Additionally, if we consider a subsystem consisting of two particles, $i$ and $j$, the quantity

$$
\begin{aligned}
\Sigma_{i j} & =\frac{1}{\theta_{i}}\left(\dot{u}_{i}-\dot{w}_{i}-\boldsymbol{\mu}_{i}^{T} \sum_{\substack{k \neq i \\
k \neq j}} \boldsymbol{J}_{i k}-\sum_{\substack{k \neq i \\
k \neq j}} R_{i k}\right) \\
& +\frac{1}{\theta_{j}}\left(\dot{u}_{j}-\dot{w}_{j}-\boldsymbol{\mu}_{j}^{T} \sum_{\substack{l \neq i \\
l \neq j}} \boldsymbol{J}_{j l}-\sum_{\substack{l \neq i \\
l \neq j}} R_{j l}\right) \\
& =\frac{\boldsymbol{\mu}_{i}^{T} \boldsymbol{J}_{i j}}{\theta_{i}}+\frac{\boldsymbol{\mu}_{j}^{T} \boldsymbol{J}_{j i}}{\theta_{j}}+\frac{R_{i j}}{\theta_{i}}+\frac{R_{j i}}{\theta_{j}}=\boldsymbol{K}_{i j}^{T} \boldsymbol{J}_{i j}+P_{i j} R_{i j},
\end{aligned}
$$

where we write

$$
\begin{aligned}
& \boldsymbol{K}_{i j}=\frac{\boldsymbol{\mu}_{i}}{\theta_{i}}-\frac{\boldsymbol{\mu}_{j}}{\theta_{j}}=k_{B}\left(\gamma_{i}-\gamma_{j}\right), \\
& P_{i j}=\frac{1}{\theta_{i}}-\frac{1}{\theta_{j}}=k_{B}\left(\beta_{i}-\beta_{j}\right),
\end{aligned}
$$

measures the internal entropy production rate for the particle pair, i. e., the entropy rate within the pair in excess of the entropy rate due to the external supply of heat. We now postulate, by way of working hypothesis, the local internal entropy production inequality, or local dissipation inequality,

$$
\Sigma_{i j} \geq 0
$$

The relation (3.11) mirrors the classical Clausius-Duhem dissipation inequality of continuum thermodynamics (cf., e. g., Malvern (1969)).

\subsection{Onsager kinetics}

Evidently, the local dissipation inequality (3.11) restricts the type of processes, or evolutions, of the temperature and atomic fraction fields that can occur. In addition, the inequality (3.11) establishes the duality pairings between thermodynamic fluxes and forces: the heat fluxes $R_{i j}$ are driven by the dual discrete temperature gradients $P_{i j}$, and the mass fluxes $\boldsymbol{J}_{i j}$ are driven by the dual discrete chemical-potential gradients $\boldsymbol{K}_{i j}$. Thus, by analogy with Onsager's theory of kinetic relations (De Groot and Mazur, 1962), the local 
dissipation inequality (3.11) suggests kinetic laws of the general form

$$
\begin{aligned}
& R_{i j}=\frac{\partial \psi}{\partial P_{i j}}(\{P\},\{\boldsymbol{K}\}), \\
& \boldsymbol{J}_{i j}=-\frac{\partial \psi}{\partial \boldsymbol{K}_{i j}}(\{P\},\{\boldsymbol{K}\}),
\end{aligned}
$$

where $\psi(\{P\},\{\boldsymbol{K}\})$ is a discrete kinetic potential. We may regard (3.12a) as a discrete Fourier law of heat conduction and (3.12b) as a discrete Fick law of diffusion. We note that relations (3.12) allow for the processes of heat and mass transport to be coupled.

Suppose, in particular, that $(\{R\},\{\boldsymbol{J}\})=(\{0\},\{\boldsymbol{0}\})$ if $(\{P\},\{\boldsymbol{K}\})=$ $(\{0\},\{\mathbf{0}\})$. Then, the local dissipation inequality (3.11) holds if and only if the kinetic potential $\psi$ is convex and we may introduce the dual kinetic potential

$$
\psi^{*}(\{R\},\{\boldsymbol{J}\})=\sup _{\{P\},\{\boldsymbol{K}\}}\left\{\{R\}^{T}\{P\}+\{\boldsymbol{J}\}^{T}\{\boldsymbol{K}\}-\psi(\{P\},\{\boldsymbol{K}\})\right\},
$$

with the properties

$$
\begin{aligned}
P_{i j} & =\frac{\partial \psi^{*}}{\partial R_{i j}}(\{R\},\{\boldsymbol{J}\}), \\
\boldsymbol{K}_{i j} & =-\frac{\partial \psi^{*}}{\partial \boldsymbol{J}_{i j}}(\{R\},\{\boldsymbol{J}\}),
\end{aligned}
$$

which effectively invert (3.12).

The discrete equations of conservation of mass and energy, (3.6) and (3.2), respectively, the latter with a heat flux of the form (3.4), may now be combined with the kinetic equations (3.12) to obtain

$$
\begin{aligned}
\frac{\dot{s}_{i}}{k_{B} \beta_{i}} & =\sum_{j \neq i} \frac{\partial \psi}{\partial P_{i j}}(\{P\},\{\boldsymbol{K}\}), \\
\dot{\boldsymbol{x}}_{i} & =-\sum_{j \neq i} \frac{\partial \psi}{\partial \boldsymbol{K}_{i j}}(\{P\},\{\boldsymbol{K}\}),
\end{aligned}
$$

which govern the evolution of the system. In particular, Eq. (3.15a) may be regarded as a discrete heat equation in entropy form, whereas Eq. (3.15b) may be regarded as a discrete mass-diffusion equation. 


\subsection{Linear kinetics}

In this work, the kinetic relations are regarded as empirical relations and, as such, subject to modeling, in the entirely same manner as empirical interatomic potentials are the subject of modeling in the field of molecular dynamics. The modeling task may be simplified by recourse to a number of ancillary modeling assumptions, such as the assumption of linear kinetics, i. e., a linear relation (3.12) between thermodynamic fluxes and driving forces.

In order to obtain a simple working kinetic model, we may further assume that heat conduction and mass transport are uncoupled, and that the kinetic potential has a bondwise structure, i. e., is the sum of contributions from particle bonds. These modeling assumptions lead to a kinetic potential of the general form

$$
\psi(\{P\},\{\boldsymbol{K}\})=\sum_{\langle i, j\rangle} \frac{1}{2} A_{i j} \theta_{i j}^{2} P_{i j}^{2}+\sum_{l=1}^{M} \sum_{\langle i, j\rangle} \frac{1}{2} B_{i j}^{l} x_{i j}^{l}\left(K_{i j}^{l}\right)^{2},
$$

where $\langle i, j\rangle$ denotes the bond defined by particles $i$ and $j$ and the sum extends over all bonds in the system, and we introduce the average bondwise temperature and atomic fractions

$$
\begin{aligned}
\theta_{i j} & =\frac{1}{2}\left(\theta_{i}+\theta_{j}\right), \\
x_{i j}^{l} & =\frac{1}{2}\left(x_{i}^{l}+x_{j}^{l}\right) .
\end{aligned}
$$

The coefficients $A_{i j}$ and $B_{i j}^{l}$ are-possibly temperature and atomic-fraction dependent - bondwise kinetic coefficients, or transport constants, to be identified for specific material systems. Within the framework of linear kinetics, couplings between the kinetic processes can be accounted for simply by allowing cross terms between $P_{i j}$ and $K_{i j}^{l}$ in (3.16), but such extensions will not be pursued here in the interest of simplicity. For the uncoupled, linearkinetics potential (3.16), the discrete kinetic equations (3.15) specialize to

$$
\begin{aligned}
\frac{\dot{s}_{i}}{k_{B} \beta_{i}} & =\sum_{\langle i, j\rangle} A_{i j} \theta_{i j}^{2} P_{i j}=\sum_{\langle i, j\rangle} A_{i j} \theta_{i j}^{2} k_{B}\left(\beta_{i}-\beta_{j}\right), \\
\dot{x}_{i}^{l} & =-\sum_{\langle i, j\rangle} B_{i j}^{l} x_{i j}^{l} K_{i j}^{l}=-\sum_{\langle i, j\rangle} B_{i j}^{l} x_{i j}^{l} k_{B}\left(\gamma_{i}^{l}-\gamma_{j}^{l}\right) .
\end{aligned}
$$


As before, we may regard (3.18a) as a discrete linear Fourier law of heat conduction and (3.18b) as a discrete linear Fick's law of diffusion.

Experimental measurements of transport properties, such as thermal expansion coefficients and mass diffusivities, supply useful constraints on the values of the bondwise kinetic coefficients. This connection can be effected by considering the long-wavelength limit of the kinetic potential (3.16). Indeed, for some simple geometries the long-wavelength limit suffices to determine all the atomic-level empirical constants. The long-wavelength limit of the discrete kinetic equations may be derived by means of a standard formalasymptotic analysis (cf., e. g., Askar (1986)), presented in Appendix A. The resulting discrete-to-continuum relations are

$$
\begin{aligned}
& -\rho c_{v} \boldsymbol{v}_{i}+\nabla \cdot \boldsymbol{\kappa}_{i}=\sum_{\langle i, j\rangle} A_{i j} \boldsymbol{r}_{j i}, \\
& \boldsymbol{\kappa}_{i}=\sum_{\langle i, j\rangle} \frac{1}{2} A_{i j} \boldsymbol{r}_{j i} \otimes \boldsymbol{r}_{j i},
\end{aligned}
$$

for heat conduction and

$$
\begin{aligned}
& \boldsymbol{v}_{i}^{l}=-\sum_{\langle i, j\rangle} k_{B} B_{i j}^{l} \boldsymbol{r}_{j i}, \\
& \boldsymbol{D}_{i}^{l}=\sum_{\langle i, j\rangle} \frac{k_{B}}{2} B_{i j}^{l}\left(\boldsymbol{r}_{j i} \otimes \boldsymbol{r}_{j i}\right),
\end{aligned}
$$

for mass transport, where $\boldsymbol{r}_{j i}$ the relative position vector between particles $i$ and $j, \rho$ is the mass density, $c_{v}$ is the heat capacity per unit mass at constant volume, $\boldsymbol{v}$ is an advection velocity for heat, $\boldsymbol{\kappa}$ is the - possibly anisotropicthermal-conductivity tensor, $\boldsymbol{v}_{l}$ is a drift velocity for species $l$ and $\boldsymbol{D}^{l}$ is the - possibly anisotropic - diffusivity tensor of species $l$.

Evidently, the drift velocities $\boldsymbol{v}_{i}$ and $\boldsymbol{v}_{i}^{l}$ vanish if the system is centrosymmetric. We also observe that the thermal conductivity tensor $\boldsymbol{\kappa}$ and the diffusivity tensors $\boldsymbol{D}_{i}^{l}$ are symmetric and, therefore, satisfy the Onsager-Casimir relations (Casimir, 1945). For disordered systems, the microscopic drift velocities and diffusivity tensors just defined may be expected to fluctuate on the atomistic scale. The macroscopic drift velocities and diffusivity tensors may then be identified with local averages of the corresponding microscopic fields over neighborhoods of size intermediate between atomistic and macroscopic. For ordered systems, such as lattices, we may expect the local drift 
velocities and diffusivity tensors to be independent of site $i$ and, hence, directly equal to the corresponding macroscopic values. For such systems and for sufficiently simple kinetic models, e. g., involving nearest-neighbor interactions, the discrete-to-continuum relations (3.19) and (3.20) may suffice to identify all the atomistic transport coefficients from experimentally measured diffusivity values.

\section{Meanfield approximation theory}

Despite the formal simplicity of the non-equilibrium statistical mechanics framework just outlined, the calculation of the thermodynamic potentials in closed form is generally intractable, which raises the need for approximation theory. In this section, we extend the classical variational meanfield theory (cf., e. g., Yeomans (1992)) to systems away from equilibrium as described by the max-ent framework developed in the foregoing. The resulting variational framework provides a convenient basis for the formulation of computationally tractable models.

\subsection{Variational meanfield theory}

A variational meanfield theory may be formulated directly in terms of the principle of maximum entropy. Thus, let $\mathcal{P}_{0}$ be a class of trial probability density functions, possibly defined parametrically. We may then identify the best probability density function $p_{0}^{*}$ in the class directly by recourse to the max-ent principle, namely,

$$
\mathcal{L}\left[p_{0}^{*},\{\beta\},\{\gamma\}\right]=\sup _{p_{0} \in \mathcal{P}_{0}} \mathcal{L}\left[p_{0},\{\beta\},\{\gamma\}\right]
$$

for fixed $\{\beta\}$ and $\{\gamma\}$. In practice, the class $\mathcal{P}_{0}$ is often chosen to consist of trial probability density functions of the form

$$
p_{0}=\frac{1}{\Xi_{0}} \mathrm{e}^{-\{\beta\}^{T}\left\{h_{0}\right\}+\{\boldsymbol{\gamma}\}^{T}\{\boldsymbol{n}\}},
$$

with

$$
\Xi_{0}=\sum_{\{\boldsymbol{n}\} \in \mathcal{O}_{N M}} \frac{1}{h^{3 N}} \int_{\Gamma} \mathrm{e}^{-\{\beta\}^{T}\left\{h_{0}\right\}+\{\boldsymbol{\gamma}\}^{T}\{\boldsymbol{n}\}} d q d p,
$$

and $\left\{h_{0}\right\}$ in some class $\mathcal{H}_{0}$ of local trial Hamiltonians, possibly defined para-

metrically. Then, the maximum-entropy principle (4.1) is equivalent to the 
minimality of the functional

$$
\mathcal{F}\left[\left\{h_{0}\right\},\{\beta\},\{\gamma\}\right]=k_{B}\{\beta\}^{T}\left\{\left\langle h-h_{0}\right\rangle_{0}\right\}-\Phi_{0}
$$

over $\mathcal{H}_{0}$, with $\Phi_{0}=k_{B} \log \Xi_{0}$, as shown next.

Proposition 4.1. Let $\mathcal{H}_{0}$ be a class of local trial Hamiltonians and $\mathcal{P}_{0}$ the corresponding class of trial probability functions of the form (4.2) and (4.3). Suppose that the functional $\mathcal{F}$ and the Lagrangian $\mathcal{L}$ attain a unique minimum and maximum at $\left\{h_{0}^{*}\right\}$ and $p_{0}^{*}$ over $\mathcal{H}_{0}$ and $\mathcal{P}_{0}$, respectively, at fixed $\{\beta\}$ and $\{\gamma\}$. Then,

$$
\mathcal{L}\left[p_{0}^{*},\{\beta\},\{\boldsymbol{\gamma}\}\right]=-\mathcal{F}\left[\left\{h_{0}^{*}\right\},\{\beta\},\{\boldsymbol{\gamma}\}\right]
$$

and $\left\{h_{0}^{*}\right\}$ and $p_{0}^{*}$ are related through (4.2) and (4.3).

Proof. Inserting (4.2) and (4.3) into the Lagrangian (2.12) gives

$$
\begin{aligned}
\mathcal{L}\left[p_{0},\{\beta\},\{\boldsymbol{\gamma}\}\right] & =-k_{B}\left\langle\log p_{0}\right\rangle_{0}-k_{B}\{\beta\}^{T}\left\{\langle h\rangle_{0}\right\}+k_{B}\{\boldsymbol{\gamma}\}^{T}\left\{\langle\boldsymbol{n}\rangle_{0}\right\} \\
& =k_{B} \log \Xi_{0}(\{\beta\},\{\boldsymbol{\gamma}\})-k_{B}\{\beta\}^{T}\left\{\left\langle h-h_{0}\right\rangle_{0}\right\} \\
& =-\mathcal{F}\left[\left\{h_{0}\right\},\{\beta\},\{\boldsymbol{\gamma}\}\right] .
\end{aligned}
$$

Hence, the maxima of $\mathcal{L}\left[p_{0},\{\beta\},\{\boldsymbol{\gamma}\}\right]$ correspond to the negative minima of $\mathcal{F}\left[\left\{h_{0}\right\},\{\beta\},\{\gamma\}\right]$, and the corresponding values satisfy relation (4.5) and are related by (4.2) and (4.3).

We note that the minimality of $\mathcal{F}$ reduces to Bogoliubov's principle (Callen, 1985; Yeomans, 1992) at equilibrium, i. e., for uniform temperature and chemical potential fields, and that it may be thus regarded as an extension of classical variational meanfield theory to systems away from equilibrium. We recall that the classical Bogoliubov principle characterizes the free energy of a system as the minimum of a functional defined on a space of trial Hamiltonians. We show next that the maximization of (4.1) admits a similar interpretation in terms of the grand-canonical free entropy of the system.

Proposition 4.2 (Maximum principle for the grand-canonical free entropy). Let $\mathcal{H}_{0}$ be a class of local trial Hamiltonians. Then,

$$
\Phi(\{\beta\},\{\gamma\}) \geq-\inf _{\left\{h_{0}\right\} \in \mathcal{H}_{0}} \mathcal{F}\left[\left\{h_{0}\right\},\{\beta\},\{\gamma\}\right]
$$

and the equality is attained for $\mathcal{H}_{0}=\mathcal{H}$. 
Proof. We have

$$
\begin{aligned}
\Phi(\{\beta\},\{\gamma\}) & =\sup _{p} \mathcal{L}[p,\{\beta\},\{\gamma\}] \geq \sup _{p_{0} \in \mathcal{P}_{0}} \mathcal{L}\left[p_{0},\{\beta\},\{\gamma\}\right] \\
& =\sup _{\left\{h_{0}\right\} \in \mathcal{H}_{0}}\left(k_{B} \log \Xi_{0}-k_{B}\{\beta\}^{T}\left\{\left\langle h-h_{0}\right\rangle_{0}\right\}\right) \\
& =-\inf _{\left\{h_{0}\right\} \in \mathcal{H}_{0}} \mathcal{F}\left[\left\{h_{0}\right\},\{\beta\},\{\gamma\}\right],
\end{aligned}
$$

with the equality attained for $\mathcal{H}_{0}=\mathcal{H}$, as advertised.

The functional $\mathcal{F}$ is well-suited to processes in which $\{\beta\}$ and $\{\gamma\}$ are the natural thermodynamic variables. Alternative functionals depending on other choices of variables may be obtained by performing appropriate Legendre transforms. For example, in processes where the controlled variables are $\{\beta\}$ and $\{\boldsymbol{x}\}$, we may define the functional

$$
\mathcal{F}^{*}\left(\left\{h_{0}\right\},\{\beta\},\{\boldsymbol{x}\}\right)=\min _{\{\gamma\}}\left(\mathcal{F}\left(\left\{h_{0}\right\},\{\beta\},\{\boldsymbol{\gamma}\}\right)+k_{B}\{\boldsymbol{\gamma}\}^{T}\{\boldsymbol{x}\}\right)
$$

By the properties of the Legendre transform, the next optimization principle follows:

Proposition 4.3 (Maximum principle for the dual grand-canonical free entropy). Let $\mathcal{H}_{0}$ be a class of local trial Hamiltonians. Then,

$$
\Phi^{*}(\{\beta\},\{\boldsymbol{x}\}) \geq-\inf _{\left\{h_{0}\right\} \in \mathcal{H}_{0}} \mathcal{F}^{*}\left[\left\{h_{0}\right\},\{\beta\},\{\boldsymbol{x}\}\right]
$$

where $\Phi^{*}=\Phi-k_{B}\{\boldsymbol{\gamma}\}^{T}\{\boldsymbol{x}\}$, with $\{\boldsymbol{x}\}$ and $\{\boldsymbol{\gamma}\}$ related by the local equilibrium relations (2.21). Moreover, the equality is attained for $\mathcal{H}_{0}=\mathcal{H}$.

We remark that the fundamental meanfield variational principles stated in Propositions 4.2 and 4.3 are formulated in terms of Massieu functions (Callen, 1985), in contrast to the classical Bogoliubov theorem, which is formulated in terms of the free energy. In general, we may expect variational meanfield theory to be increasingly accurate as the space of trial Hamiltonians is increased. The particular space to be used in practice is a compromise between accuracy and computational tractability.

A powerful connection between variational meanfield theory and variational time-integrators (Ortiz and Stainier, 1999) may be forged by recourse to time discretization (Yang et al., 2006; Kulkarni et al., 2008; Ponga et al., 
2012). It is indeed possible to formulate time-discretized minimum principles that fold in both the time-discretization and meanfield approximations into a single functional whose stationarity yields consistent time-discretizations of all governing equations. The same minimum principle likewise supplies a basis for spatial coarse-graining, e. g., by recourse to the quasi-continuum method (Tadmor et al., 1996; Knap and Ortiz, 2001). However, these applications of the variational framework are beyond the scope of the present paper and will not be pursued here.

\section{Comparison with experiment: Equilibrium properties}

Since the feasibility of the approach formulated in the foregoing hinges critically on the formulation of computationally tractable meanfield approximations, we proceed to assess the fidelity of such meanfield models by direct comparison with experimental observation. To this end, we formulate a simple meanfield model for substitutional alloys and assess its ability to reproduce equilibrium properties of four alloys, including lattice parameter, linear thermal expansion coefficient, elastic moduli and surface segregation concentration.

\subsection{A simple meanfield model}

A simple meanfield model for substitutional alloys is obtained by considering local trial Hamiltonians of the form

$$
h_{0 i}(\{\boldsymbol{q}\},\{\boldsymbol{p}\},\{\boldsymbol{n}\})=\frac{1}{2 m\left(\boldsymbol{n}_{i}\right)}\left|\boldsymbol{p}_{i}-\overline{\boldsymbol{p}}_{i}\right|^{2}+\frac{m\left(\boldsymbol{n}_{i}\right) \omega_{i}^{2}}{2}\left|\boldsymbol{q}_{i}-\overline{\boldsymbol{q}}_{i}\right|^{2}
$$

where

$$
\frac{1}{m\left(\boldsymbol{n}_{\boldsymbol{i}}\right)}=\sum_{k=1}^{M} \frac{n_{i k}}{m_{k}}
$$

is the mass of site $i$ defined in terms of the atomic mass $m_{k}$ of species $k$. The local trial Hamiltonians (5.1), which generalize the local trial Hamiltonians proposed by Kulkarni et al. (2008) to alloys, are parameterized by the fields $\{\overline{\boldsymbol{q}}\}=\left(\overline{\boldsymbol{q}}_{i}\right)_{i=1}^{N},\{\overline{\boldsymbol{p}}\}=\left(\overline{\boldsymbol{p}}_{i}\right)_{i=1}^{N}$ and $\{\omega\}=\left(\omega_{i}\right)_{i=1}^{N}$, which may be regarded as the mean position and momenta of the particles and their effective frequency of vibration, respectively. The optimal value of these fields follows variationally by maximization of the entropy functional (4.1) or, alternatively, by minimization of the energy functional (4.4). We also note 
that local trial Hamiltonians are chosen to be quadratic and to depend on the local position and momentum of the particle only. However, it should be carefully noted that the meanfield model accounts for interatomic interactions and anharmonicity through the functional (4.4), which involves the full local Hamiltonians.

\subsection{Meanfield entropy and driving forces}

For trial local Hamiltonians of the form (5.1), a straightforward calculation gives (4.3) and (4.1) as

$$
\Xi_{0}=\prod_{i=1}^{N} \frac{\sum_{k=1}^{M} \mathrm{e}^{\gamma_{i k}}}{\left(\hbar \beta_{i} \omega_{i}\right)^{3}}
$$

and

$$
p_{0}(\{\boldsymbol{q}\},\{\boldsymbol{p}\},\{\boldsymbol{n}\})=\prod_{i=1}^{N} \frac{\mathrm{e}^{-\beta_{i} h_{0 i}}}{\left(\hbar \beta_{i} \omega_{i}\right)^{-3}} \frac{\mathrm{e}^{\boldsymbol{\gamma}_{i}^{T} \boldsymbol{n}_{i}}}{\sum_{k=1}^{M} \mathrm{e}^{\gamma_{i k}}},
$$

respectively, where the reduced Planck constant $\hbar=h / 2 \pi$ has been introduced for normalization purposes. In addition, the average meanfield energy can be explicitly calculated, with the result

$$
\left\langle h_{0 i}\right\rangle_{0}=\frac{3}{\beta_{i}},
$$

which identifies $\beta_{i}^{-1}$ or, equivalently, $k_{B} \theta_{i}$, as the average meanfield energy per degree of freedom of atom $i$. Finally, the expected atomic fractions follow as

$$
x_{i k}=\left\langle n_{i k}\right\rangle_{0}=\frac{\mathrm{e}^{\gamma_{i k}}}{\sum_{l=1}^{M} \mathrm{e}^{\gamma_{i l}}},
$$

which can be inverted, making use of equation (2.13), to give

$$
\gamma_{i k}=-\log x_{i k}+\frac{1}{M} \sum_{l=1}^{M} \log x_{i l} .
$$

Inserting the the preceding expressions into the functionals (4.4) and (4.9) we obtain, explicitly,

$$
\begin{aligned}
& \mathcal{F}\left[\left\{h_{0}\right\},\{\beta\},\{\gamma\}\right]= \\
& k_{B} \sum_{i=1}^{N}\left[\beta_{i}\left\langle h_{i}\right\rangle_{0}-3+3 \log \left(\hbar \beta_{i} \omega_{i}\right)-\log \left(\sum_{k=1}^{M} \mathrm{e}^{\gamma_{i k}}\right)\right],
\end{aligned}
$$


and

$$
\begin{aligned}
& \mathcal{F}^{*}\left[\left\{h_{0}\right\},\{\beta\},\{\boldsymbol{x}\}\right]= \\
& k_{B} \sum_{i=1}^{N}\left[\beta_{i}\left\langle h_{i}\right\rangle_{0}-3+3 \log \left(\hbar \beta_{i} \omega_{i}\right)+\sum_{k=1}^{M} x_{i k} \log x_{i k}\right],
\end{aligned}
$$

respectively. Under quasistatic conditions, the optimal values $\left\{\overline{\boldsymbol{q}}^{*}\right\},\left\{\overline{\boldsymbol{p}}^{*}\right\}$ and $\left\{\omega^{*}\right\}$, of the atomic positions, momenta and frequencies now follow from the maximum grand-canonical free-entropy principle, eq. (4.7), as

$$
\left(\left\{\overline{\boldsymbol{q}}^{*}\right\},\left\{\overline{\boldsymbol{p}}^{*}\right\},\left\{\omega^{*}\right\}\right) \in \operatorname{argmin} \mathcal{F}
$$

at constant $\{\beta\}$ and $\{\gamma\}$. The corresponding Euler-Lagrange equations are

$$
\begin{aligned}
& \frac{\partial \mathcal{F}}{\partial \overline{\boldsymbol{q}}_{i}}=\frac{\partial}{\partial \overline{\boldsymbol{q}}_{i}}\left(k_{B}\{\beta\}^{T}\left\{\langle h\rangle_{0}\right\}\right)=0 \\
& \frac{\partial \mathcal{F}}{\partial \overline{\boldsymbol{p}}_{i}}=\frac{\partial}{\partial \overline{\boldsymbol{p}}_{i}}\left(k_{B}\{\beta\}^{T}\left\{\langle h\rangle_{0}\right\}\right)=0 \\
& \frac{\partial \mathcal{F}}{\partial \omega_{i}}=\frac{\partial}{\partial \omega_{i}}\left(k_{B}\{\beta\}^{T}\left\{\langle h\rangle_{0}\right\}\right)+\frac{3 k_{B}}{\omega_{i}}=0
\end{aligned}
$$

These equations define a highly coupled system of equations in the unknowns $(\{\overline{\boldsymbol{q}}\},\{\overline{\boldsymbol{p}}\},\{\omega\})$. We may regard the first two of these equations as the meanfield conditions of quasistatic equilibrium for the mean atomic positions and momenta $\{\overline{\boldsymbol{q}}\},\{\overline{\boldsymbol{p}}\}$, respectively, whereas the third may be regarded as determining the mean atomic frequencies $\{\omega\}$ that best represent the Hamiltonian $H$ of the system in a variational meanfield sense.

\subsection{Evaluation of meanfield phase averages}

As is evident from (5.8) and (5.9), the evaluation of the functionals $\mathcal{F}$ and $\mathcal{F}^{*}$ requires the calculation of the effective local Hamiltonians $\left\langle h_{i}\right\rangle_{0}$, i. e., the phase average of the local Hamiltonians with respect to the meanfield probability density (5.4). We note that $\langle h\rangle_{0}$ depends not only on the mean atomic positions $\{\overline{\boldsymbol{q}}\}$ and momenta $\{\overline{\boldsymbol{p}}\}$, but also on the variables $\{\omega\},\{\beta\}$ and $\{\gamma\}$. If, for instance, the local Hamiltonians are of the form

$$
h_{i}(\{\boldsymbol{q}\},\{\boldsymbol{p}\},\{\boldsymbol{n}\})=\frac{1}{2 m\left(\boldsymbol{n}_{i}\right)}\left|\boldsymbol{p}_{i}\right|^{2}+V_{i}(\{\boldsymbol{q}\},\{\boldsymbol{n}\}),
$$


where $V_{i}(\{\boldsymbol{q}\},\{\boldsymbol{n}\})$ is a multi-species interatomic potential, then we have

$$
\left\langle h_{i}\right\rangle_{0}=\frac{3}{2 \beta_{i}}+\frac{1}{2 \bar{m}\left(\boldsymbol{x}_{i}\right)}\left|\overline{\boldsymbol{p}}_{i}\right|^{2}+\left\langle V_{i}\right\rangle_{0},
$$

where

$$
\frac{1}{\bar{m}\left(\boldsymbol{x}_{i}\right)}=\left\langle\sum_{k=1}^{M} \frac{n_{i k}}{m_{k}}\right\rangle_{0}=\sum_{k=1}^{M} \frac{x_{i k}}{m_{k}} .
$$

In this expression, $\bar{m}\left(\boldsymbol{x}_{i}\right)$ is the effective mass of particle $i$, which can alternatively be expressed as a function of $\gamma_{i}$ using relation (5.6) and $\left\langle V_{i}\right\rangle_{0}$ is an effective interatomic potential, both of which depend on local temperature and stoichiometry in general. We note that the meanfield averaging $\left\langle V_{i}\right\rangle_{0}$ with respect to the occupation numbers $\{\boldsymbol{n}\}$ can be carried out explicitly. We also note that the meanfield probability distribution (5.4) is expressed in terms Gaussian functions. By virtue of this crucial feature, the mean field averaging $\left\langle V_{i}\right\rangle_{0}$ with respect to atomic coordinates can be conveniently computed numerically using Gaussian quadrature (Kulkarni et al., 2008). In the present work, all phase averages are computed using third-order Gaussianquadrature rules (Stroud (1971)).

\subsection{Embedded-Atom Method potentials}

We further illustrate the structure of the resulting effective interatomic potentials by considering empirical many-body potentials of the Embedded Atom Method (EAM) type (Ackland and Vitek (1990); Johnson (1989a); Foiles et al. (1986)). The classical EAM potentials take the form

$$
V_{i}(\{\boldsymbol{q}\})=F\left(\rho_{i}\right)+\sum_{\substack{j=1 \\ j \neq i}}^{N} \Phi\left(r_{i j}\right),
$$

where

$$
\rho_{i}=\sum_{\substack{j=1 \\ j \neq i}}^{N} f\left(r_{i j}\right)
$$

is the electron density felt by atom $i$ due to the environment,

$$
r_{i j}=\left|\boldsymbol{q}_{i}-\boldsymbol{q}_{j}\right|
$$


is the distance between atoms $i$ and $j, \Phi\left(r_{i j}\right)$ is a pair potential, and $F\left(\rho_{i}\right)$ is the energy of embedding atom $i$ in the local electron density $\rho_{i}$. For many materials of interest, the pair potential $\Phi\left(r_{i j}\right)$ is short-range, e. g., due to screening, and the pairwise sum in (5.15) reduces to $N$ sums over local neighborhoods of each atom. For a substitutional alloy, the EAM potential generalizes to (Johnson, 1989a)

$$
V_{i}(\{\boldsymbol{q}\},\{\boldsymbol{n}\})=\sum_{k=1}^{M} n_{i k} F_{k}\left(\rho_{i}\right)+\sum_{\substack{j=1 \\ j \neq i}}^{N} \sum_{k=1}^{M} \sum_{l=1}^{M} n_{i k} n_{j l} \Phi_{k l}\left(r_{i j}\right),
$$

where

$$
\rho_{i}=\sum_{\substack{j=1 \\ j \neq i}}^{N} \sum_{l=1}^{M} n_{j l} f_{l}\left(r_{i j}\right)
$$

These potentials are employed in all subsequent calculations. As already noted, the meanfield averages with respect to coordinates can be computed using Gaussian quadrature, whereas the meanfield averages with respect to occupancy numbers can be evaluated explicitly. However, the number of terms in this latter operation grows exponentially with the number of neighbors entering $V_{i}(\{\boldsymbol{q}\},\{\boldsymbol{n}\})$, which may render computations costly. Therefore, as a further approximation in calculations we set the meanfield average with respect to occupancy to

$$
\left\langle V_{i}(\{\boldsymbol{q}\}, \cdot)\right\rangle_{0} \approx \sum_{k=1}^{M} x_{i k} F_{k}\left(\bar{\rho}_{i}\right)+\sum_{\substack{j=1 \\ j \neq i}}^{N} \sum_{k=1}^{M} \sum_{l=1}^{M} x_{i k} x_{j l} \Phi_{k l}\left(r_{i j}\right),
$$

with

$$
\bar{\rho}_{i}=\sum_{\substack{j=1 \\ j \neq i}}^{N} \sum_{l=1}^{M} x_{j l} f_{l}\left(r_{i j}\right)
$$

If the embedding function $F_{k}$ is convex (respectively, concave), then this approximation gives a lower bound (respectively, upper bound) on the effective interatomic potential by Jensen's inequality.

\subsection{Nickel-Palladium Lattice Parameter}

Nickel $(\mathrm{Ni})$ and palladium $(\mathrm{Pd})$ are fcc metals at ambient conditions and form a fcc random solid solution through the entire range of compositions 


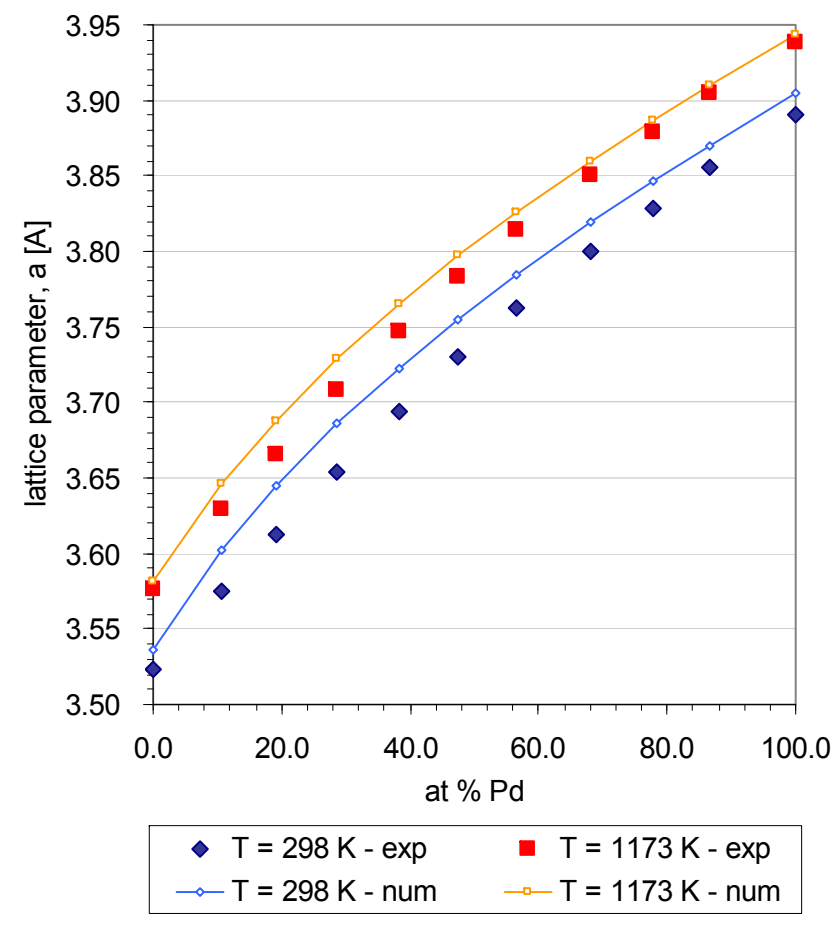

Figure 5.1: Predicted NiPd lattice parameter versus Pd atomic atomic fraction at two different temperatures. Experimental data from Bidwell (1964) also shown.

(Bidwell (1964)). We calculate the alloy lattice parameter $a$ by minimizing $\mathcal{F}^{*}$, eq. (5.9), over periodic samples containing 256 lattice sites $(4 \times 4 \times 4)$ unit cells) and over a range of uniform temperatures and $\mathrm{Pd}$ concentrations. Specifically, $\mathcal{F}^{*}$ is minimized with respect to atomic positions $\overline{\boldsymbol{q}}$ and frequencies $\{\omega\}$ at prescribed values of temperature $T$ and $\mathrm{Pd}$ atomic fraction $\sim x_{A}$ and the equilibrium lattice parameter $a$ is determined from the relaxed configuration of the sample.

Fig. 5.1 shows a comparison between lattice parameters thus computed and single-crystal measurements at two different temperatures (Bidwell, 1964). Both predicted and experimental values vary smoothly with composition, with a considerable overshoot of Vergard's law, namely, $a_{A B}=x_{A} a_{A}+(1-$ $\left.x_{A}\right) a_{B}$, where $a_{A B}$ is the alloy lattice parameter and $a_{A}$ and $a_{B}$ are the lattice parameters of the alloy components. The good agreement between the meanfield predictions and observation demonstrates the ability of the model 
to capture deviations from Vergard's law.

\subsection{Copper-Nickel Thermal Expansion}

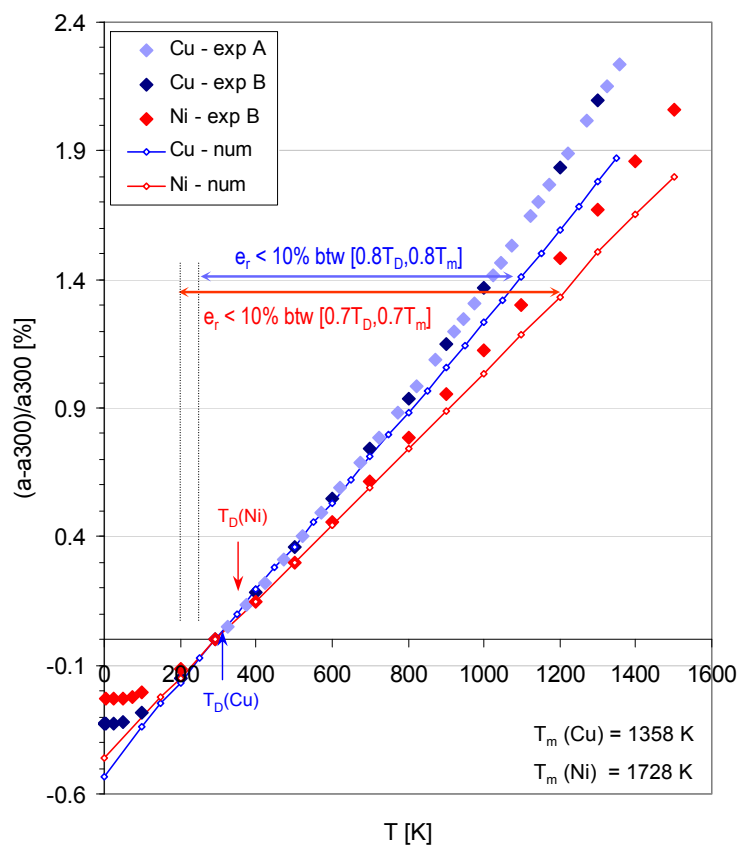

Figure 5.2: Predicted and measured coefficients of thermal expansion in CuNi single-crystal alloys as a function of temperature. Experimental data from Simmons and Balluffi (1963) (A) and Toloukian (1975) (B). Also shown for reference are the melting temperature $T_{m}$ and the Debye temperature $T_{D}$.

Copper $(\mathrm{Cu})$ and nickel $(\mathrm{Ni})$ are also fcc metals and also form a fcc random solid solution through the entire range of compositions. As previously, we calculate the alloy lattice parameter $a$ by minimizing $\mathcal{F}^{*}$, eq. (5.9), over periodic samples containing 500 lattice sites $(5 \times 5 \times 5$ unit cells $)$ and over a range of uniform temperatures and $\mathrm{Cu}$ concentrations. From the equilibrium lattice parameter $a$, we additionally compute the coefficient of thermal expansion as

$$
\alpha=\frac{a-a_{0}}{a_{0}}
$$

where $a_{0}$ is the equilibrium lattice parameter of the alloy at room temperature. 
Figure 5.2 shows a comparison between predicted and experimental values of the thermal expansion coefficient (Toloukian, 1975; Simmons and Balluffi, 1963) for two different single-crystal $\mathrm{CuNi}$ alloys as a function of temperature. The model captures the dependence of the coefficient of thermal expansion on concentration over a broad range of temperatures. The higher thermal expansion observed in Cu-rich samples is also captured by the model. This agreement notwithstanding, the figure serves to exhibit some of the limitations of the simple meanfree model under consideration. Indeed, the discrepancy between model and experiment at low temperatures is expected, as the model is predicated on classical statistical mechanics and quantum effects, which become important at low temperatures, are entirely neglected. In addition, the meanfree model underestimates two-point correlations between thermal vibrations, thus resulting in deviations from experiment at high temperatures (Kulkarni et al., 2008).

\subsection{Copper-Nickel Elastic Constants}

We determine the three independent elastic moduli of $\mathrm{CuNi}$ alloys, $C_{11}$, $C_{12}$ and $C_{44}$, by minimizing $\mathcal{F}^{*},(5.9)$, over samples containing 256 lattice sites $(4 \times 4 \times 4$ unit cells $)$ with respect to the atomic frequencies $\{\omega\}$ at room temperature and over a range of copper atomic fractions $\sim x_{\mathrm{Cu}}$ and prescribed uniform deformations at intervals of 0.0001. A fifth-order polynomial is fitted through the calculated energies whence the elastic constant are obtained by differentiation about the undeformed configuration of the alloy (cf. Venturini (2011) for additional details).

Figure 5.3 compares computed and experimental elastic moduli (Simmons, 1971) in single-crystal $\mathrm{CuNi}$ alloys at room temperature as a function of the copper concentration. The agreement is good for $C_{11}$ and the Voigtaverage shear modulus $G=\left(C_{11}-C_{12}+3 C_{44}\right) / 5$, including the dependence of the moduli on temperature, and fair for the bulk modulus $B=\left(C_{11}+2 C_{12}\right) / 3$. The systematic discrepancy in the bulk modulus may be traced to the calibration of the Johnson (1988) EAM potential, which constrains the anisotropy ratio $A=2 C_{44} /\left(C_{11}-C_{12}\right)$ to be 2 and, in consequence, can only match exactly two of the three elastic moduli of the pure phases.

\subsection{Surface Segregation}

The preceding validation cases are concerned with equilibrium bulk properties of alloys, corresponding to equilibrium configurations that are statistically uniform. By way of contrast, free surfaces supply a simple geometry 


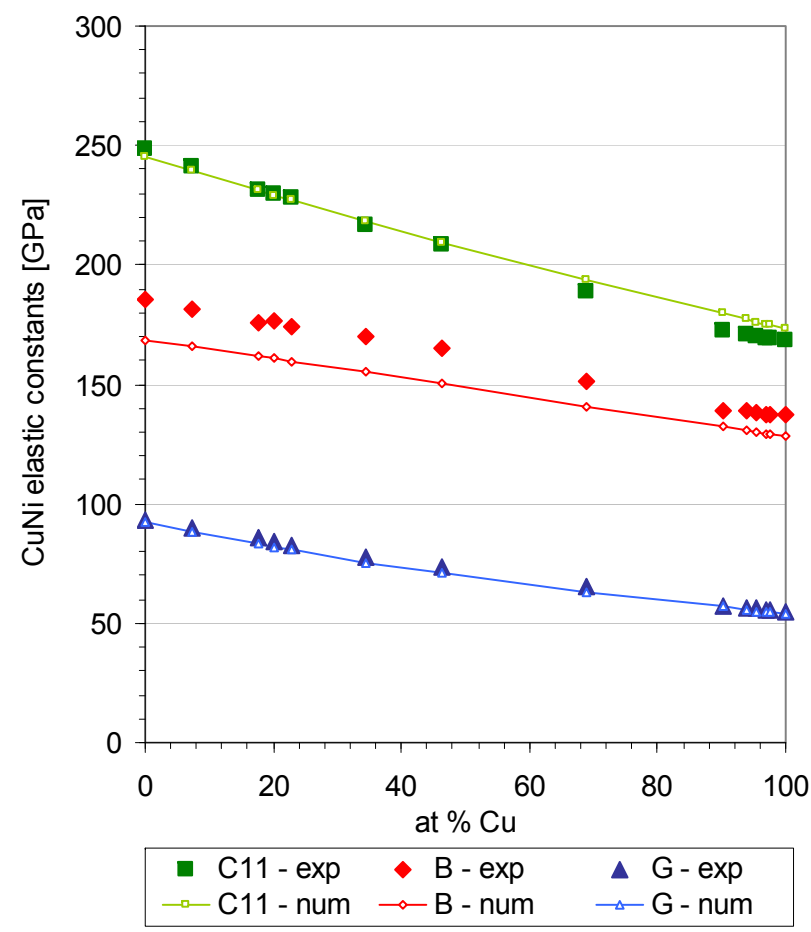

Figure 5.3: Predicted and experimental (Simmons, 1971) elastic constants in single-crystal $\mathrm{CuNi}$ alloys at room temperature as a function of $\mathrm{Cu}$ concentration.

for which the equilibrium fields are not uniform but involve segregation of the alloying species. Surface segregation owes to three main driving forces. The first driver is the lowering of the surface energy, resulting in the element with the lower surface energy segregating to the surface. This segregation is face-dependent, as the coordination number of the surface atoms depends on the orientation of the surface. By virtue of this effect, segregation in an fcc lattice is most pronounced at on (110) faces, followed by (100) faces and least pronounced on (111) faces (Bozzolo et al., 2007). The second driving force for segregation is the lowering of the total bond energy. Thus, when the $\mathrm{AB}$ bond has more energy than the average of the $\mathrm{AA}$ and $\mathrm{BB}$ bonds, there is a preference for $\mathrm{AA}$ and $\mathrm{BB}$ bonds, with the result that the attendant $\mathrm{AB}$ repulsions promote the segregation of the alloying species and give rise to a monotonic depth profile. For other orderings of the bond energies, oscillatory depth profile may arise (Bozzolo et al. (2007)). The third driving 


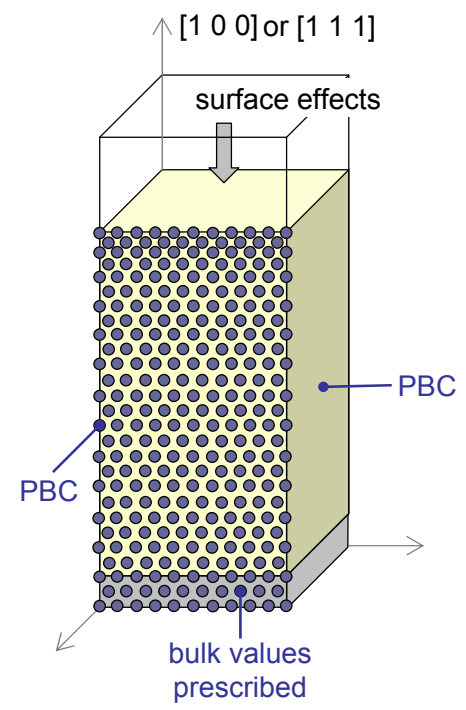

Figure 5.4: Schematic of the computational domain and boundary conditions used to simulate surface segregation.

force pertains to the relaxation of elastic strain energy due to the atomic-size contrast between A and B atoms. This effect favors the segregation of the alloying species when the contrast is large. In addition to energetic driving forces, segregation is also driven by entropic effects, since segregation to the boundary tends to lower the entropy of the system. This entropic effect tends to inhibit segregation at higher temperatures (Bozzolo et al. (2007)). Evidently, the proper accounting of these competing effects requires atomistic fidelity, including accurate multispecies potentials and entropies, and thus supplies an exacting validation of the theory.

The calculations proceed in three steps. Firstly, we evaluate the properties of a statistically uniform alloy by minimizing $\mathcal{F}^{*}$, eq. (5.9) over periodic samples containing $640(4 \times 4 \times 10)$ sites with respect to atomic positions $\overline{\boldsymbol{q}}$ and frequencies $\{\omega\}$ at fixed atomic fractions. The minimum value of $\mathcal{F}^{*}$, which equals the negative of the meanfield grand-canonical free entropy, is then differentiated numerically with respect to composition in order to obtain the corresponding chemical potentials. Finally, we introduce the appropriate free surface and minimize the functional $\mathcal{F}$ of the sample, eq. (5.8), subject to periodic boundary conditions in the directions parallel to the surface, 
Fig. 5.4. In addition, atoms at the bottom of the sample are constrained to the lattice spacing, atomic fraction and frequency of the bulk alloy at equilibrium.

\subsubsection{Copper-nickel alloys}
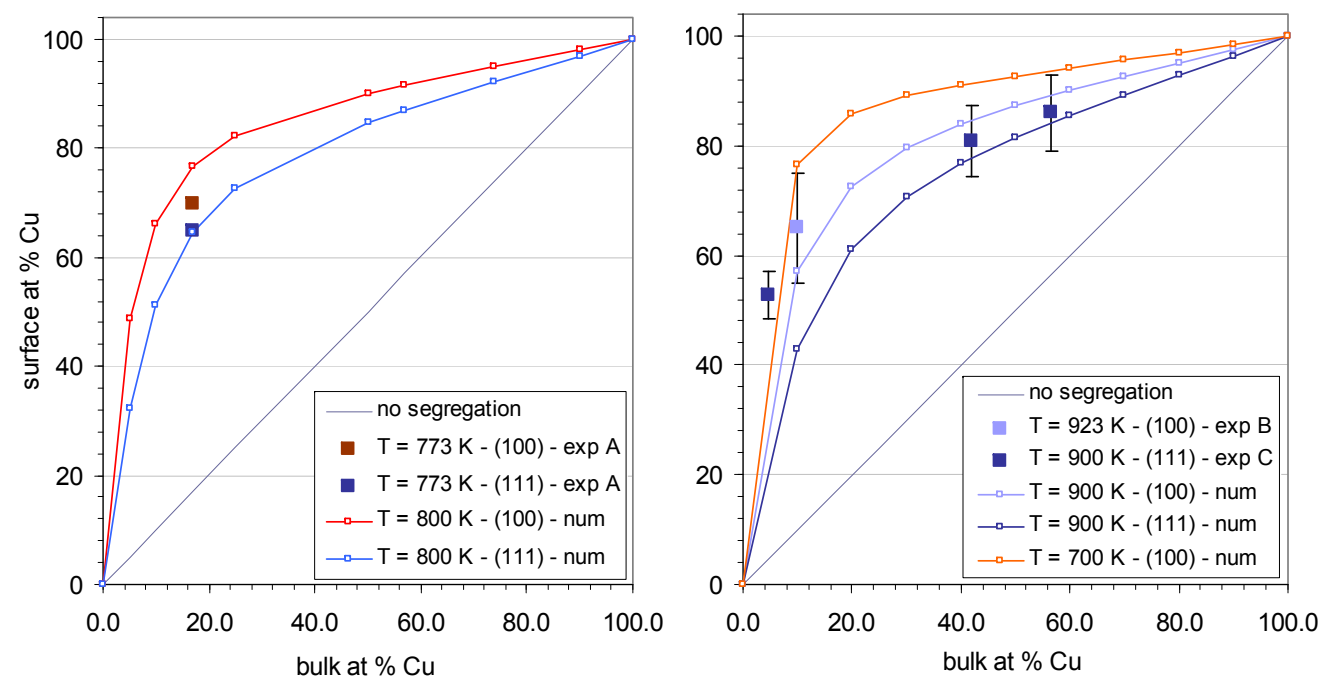

Figure 5.5: Predicted and measured surface $\mathrm{Cu}$ concentrations in $\mathrm{CuNi}$ single-crystal alloys as a function of temperature, composition and surface orientation. Experimental data from Wandelt and Brundle (1981) (A), Helms and Yu (1975) (B) and Sakurai et al. (1985) (C).

Interest in the physics and chemistry of CuNi surfaces owes to the enhancement of the selectivity and catalytic activity that these alloys exhibit over pure metals for certain reactions. For example, $\mathrm{CuNi}$ alloys are used in cyclohexane dehydrogenation into benzene (Sakurai et al., 1985). An important factors controlling those effects is the composition of the upper-most layer of the system (Helms and Yu, 1975; Sakurai et al., 1985).

Fig. 5.5 shows the computed surface $\mathrm{Cu}$ concentration $x_{\mathrm{Cu}}^{\mathrm{surf}}$ as a function of its bulk counterpart $x_{\mathrm{Cu}}^{\mathrm{bulk}}$. The figure also shows experimental measurements of Wandelt and Brundle (1981), Helms and Yu (1975) and Sakurai et al. (1985) by way of comparison. Deviations from the line $x_{\mathrm{Cu}}^{\text {surf }}=x_{\mathrm{Cu}}^{\mathrm{bulk}}$ in the plot indicate segregation. Thus, if the surface concentration is above the line, $\mathrm{Cu}$ segregates to the surface; if below, the concentration of $\mathrm{Ni}$ at 
the surface is greater than in the bulk. As may be seen from the figure, the model is able to predict the effect of temperature and crystal-face orientation on the equilibrium surface concentration. In particular, the model predicts that segregation is more favorable in open (100) surfaces. This effect may be understood by noting that the number of broken bonds is 4 for a (100) surface and 3 for the (111) surface. According to the Johnson (1989a) EAM potential, the cohesive energy of $\mathrm{Cu}$ is $3.54 \mathrm{eV}$ and $4.45 \mathrm{eV}$ for Ni. Thus, the system tends to compensate for the higher number of dangling bonds at the (100) surface by maximizing the number of low-energy $\mathrm{Cu}$ broken bonds. The model also correctly predicts that segregation is inhibited at higher temperature, a demonstration of the ability of the model to properly account for entropic effects.

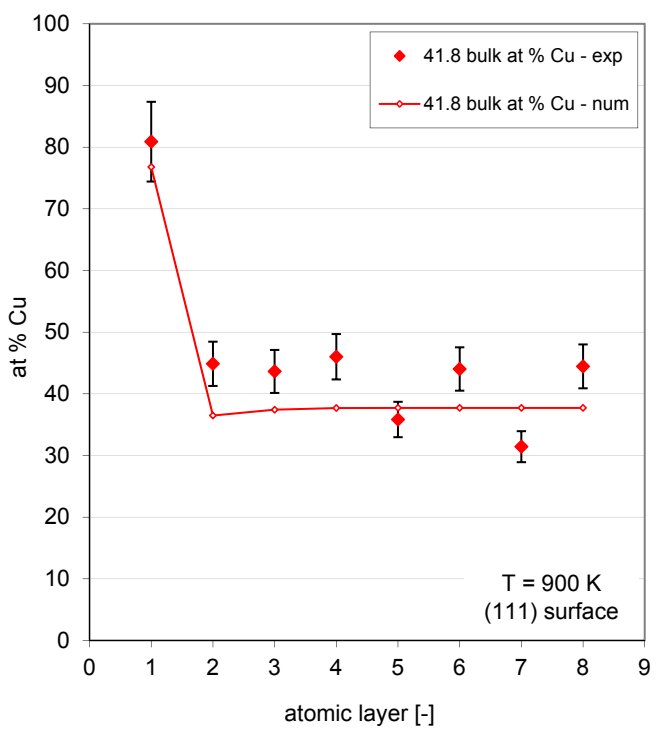

Figure 5.6: Predicted and measured surface concentration profiles of $\mathrm{Cu}$ in $\mathrm{CuNi}$ singlecrystal alloys. Experimental data from Sakurai et al. (1985).

Fig. 5.6 shows a comparison between predicted and measured (Sakurai et al., 1985) surface concentration profiles of $\mathrm{Cu}$ in $\mathrm{CuNi}$ single-crystal alloys. As may be seen from the figure, the observed monotonic $\mathrm{Cu}$ concentration profile is well-captured by the model. Such an monotonic profile is expected for the CuNi system due its positive heat of mixing (Bozzolo et al., 2007). 


\subsubsection{Gold-silver alloys}
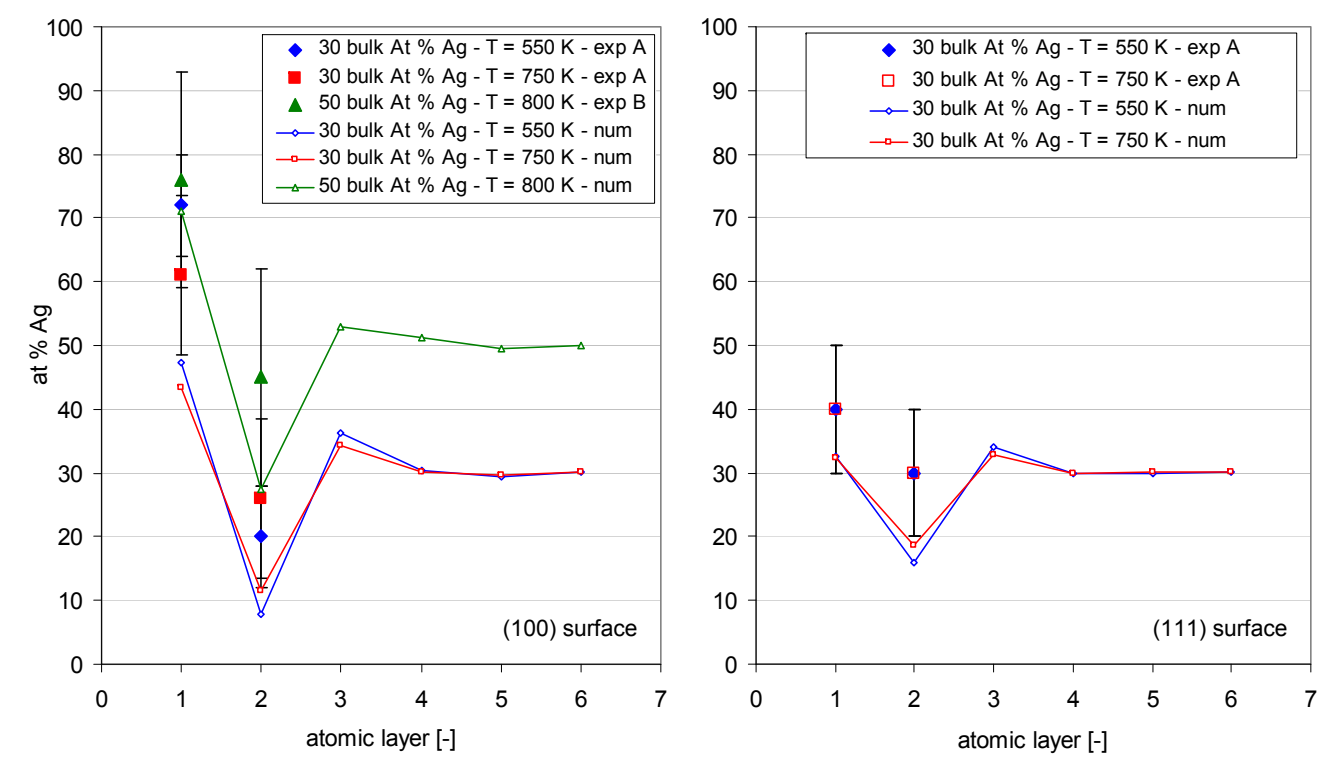

Figure 5.7: Predicted and measured surface concentrations of Ag in AuAg single-crystal alloys as a function of temperature, composition and surface orientation. Experimental data from King and Donnelly (1985) (A) and Derry and Wan (2004) (B).

The gold $(\mathrm{Au})$-silver $(\mathrm{Ag})$ system forms a disordered solid solution over the entire range of concentrations (Ackland and Vitek, 1990). Both metals and their alloys form fcc crystal structures. Fig. 5.7 shows a comparison between predicted and measured (King and Donnelly, 1985; Derry and Wan, 2004) surface segregation profiles for different surface orientations, compositions and temperatures. Remarkably, in contrast to the copper-nickel alloys considered earlier, Fig. 5.6, the Ag concentration exhibits an oscillatory profile with depth, which is captured by the model. Such an oscillatory profile is expected for the AgAu system due its negative heat of mixing (Bozzolo et al., 2007). Indeed, since the AgAu bond has less energy than the average of the $\mathrm{AgAg}$ and $\mathrm{AuAu}$ bonds, there is a preference for $\mathrm{AgAu}$ bonds within the system, with the result that, when Ag segregates to the surface, a second Au-rich layer becomes energetically favorable. The model also correctly predicts that segregation is more favorable in open (100) surfaces, which, in addition, are more sensitive to temperature. In particular, the oscillatory 
profile is more pronounced for the (100) than for the (111) surface (King and Donnelly, 1985). Finally, the model also correctly predicts that segregation is reduced with temperature, as evinced by the lower crest/valley ratio at $800 \mathrm{~K}$ in Fig. 5.7a. This prediction again provides a demonstration of the ability of the model to properly account for entropic effects.

\section{Comparison with experiment: Kinetic properties}

The validation cases presented in the preceding section have been concerned with the ability of the max-entropy formalism, presented in Section 2, in conjunction with variational meanfield approximation and standard empirical interatomic potentials, to properly account for thermodynamic driving forces and, in particular, for equilibrium properties, including both energetic and entropic effects. Such validation in turn ensures the accuracy and fidelity of the thermodynamic forces that drive kinetics away from equilibrium. A second building block of the theory, presented in Section 3, concerns the formulation of atomic-based but empirical kinetic relations of the Onsager type, with particular application to heat and mass transport. In this section, we turn attention to this latter aspect of the theory and endeavor to ascertain its validity by means of two test cases: Heat conduction in silicon nanowires and hydrogen storage in palladium. These two validation cases are next considered in turn.

\subsection{Heat conduction in Si nanowires}

Whereas bulk silicon is a poor thermoelectric material, silicon-based nanostructures in the form of one-dimensional conductors or nanowires have been extensively investigated as potentially efficient thermoelectric materials (Hicks and Dresselhaus, 1993). In particular, silicon nanowires (SiNW) have shown promise as components for nanoelectronic devices, thereby spurring extensive research into synthesis processes (Cui et al., 2001; Hochbaum et al., 2008) and fundamental thermal and electrical properties (Li et al., 2003; Shi et al., 2003; Chen et al., 2008; Doerk et al., 2010). In particular, these investigations have revealed a strong dependence of thermal conductivity on size and surface morphology (Cui et al., 2001). Thus, Cui et al. (2001) synthesized monodispersed SiNW with well-controlled diameters via a vapor-liquid-solid (VLS) mechanism using Au-nanocluster catalysts. They confirmed by high resolution TEM that using this catalyst SiNW cores remain crystalline with an amorphous surface layer of 1-3 nm thickness. Measurements of the thermal 
conductivity of SiNWs were reported by Li et al. (2003), who measured thermal conductivities more than two orders of magnitude lower than bulk from VLS-grown nanowires with relatively smooth surfaces of diameters ranging from 22 to $115 \mathrm{~nm}$. More recent works by Chen et al. (2008) and Doerk et al. (2010) have confirmed these observations and proposed explanations based on phonon-boundary scattering in thin nanowires. Simultaneously, much computational effort has been devoted to the elucidation of heat transport phenomena in very thin SiNW. Markussen et al. (2008) have computed the heat conductance for ultrathin SiNWs of diameters ranging from 1 to $5 \mathrm{~nm}$ using density-functional theory (DFT) and Tersoff empirical potential for thicker sections. They find that heat conductance of SiNWs is strongly anisotropic and that wire orientation results in variations in thermal conductance of $50 \%$ to $75 \%$. More recently, atomistic simulations carried out by Donadio and Galli (2009) suggest that the presence of a disordered surface layer as thin as two atomic distances is sufficient to reduce the thermal conductivity of SiNWs by an order of magnitude with respect to that of crystalline wires of the same radius.

These observations, data and complex phenomena pertaining to heat transport in SiNWs supply an exacting test of the ability of atomic-levelalbeit empirical - heat-conduction models to account for the anisotropy, temperature and size dependence of thermal conductivity.

\subsubsection{A simple model of heat transport in SiNWs}

We specifically aim to develop a simple model of heat transport experiments in SiNWs of Li et al. (2003). We begin by assuming that the system is rigid and devoid of mass diffusion, by virtue of which assumptions the model and the attendant simulations exercise the heat-transport kinetic relations only. As in the experiments of Li et al. (2003), we consider single-crystal SiNWs oriented in the $\langle 111\rangle$ direction, of radii $R=11,18.5,28$, and 57.5 $\mathrm{nm}$, and endeavor to determine the steady-state discrete temperature field under a prescribed temperature gradient. For each nanowire, we define the computational cell $\Omega$ to be a slice of thickness $L$ comprising 20 (111) atomic planes. We choose a Cartesian coordinate system such that its $z$ coordinate

coincides with the axis of the nanowire, Fig. 6.1. We peg the temperature of one atom to the ambient temperature, denoted $T_{0}$, and append constraints enforcing a temperature variation $\Delta T=5.0 \mathrm{~K}$ across the thickness of the slice, consistent with the experimental ambient temperature and temperature gradient. In addition, in order to terminate the computational domain 
we extend the solution outside $\Omega$ by linear extrapolation in the axial direction.

The steady-state discrete temperature field $\{\theta\}$ and macroscopic heat flux $\bar{J}_{z}$ follow from the problem

$$
\delta\left\{\sum_{\langle i, j\rangle} \frac{1}{2} A_{i j} \theta_{i j}^{2} P_{i j}^{2}-\frac{\bar{J}_{z}}{\bar{T}_{0}^{2}}\left(\sum_{i \in \Gamma_{f}} \theta_{i} \sigma_{i}-\sum_{i \in \Gamma_{l}} \theta_{i} \sigma_{i}-|\Omega| \bar{T}_{z}\right)-\lambda\left(\theta_{i_{0}}-T_{0}\right)\right\}=0,
$$

where $\theta_{i j}$ denotes the mean bond temperature, eq. (3.17a), $P_{i j}$ the discrete bondwise driving force for heat conduction, eq. (3.10b), $\Gamma_{f}$ and $\Gamma_{l}$ are the (111) planes bounding the nanowire slice,

$$
\bar{T}_{z}=\frac{\Delta T}{L}
$$

is the nominal temperature gradient, $\lambda$ is a Lagrange multiplier, $i_{0}$ is the site at which the ambient temperature $T_{0}$ is prescribed and $\Omega$ is the volume of the computational cell. In addition, $\sigma_{i}$ is a cross-sectional area of the nanowire associated with atom $i \in \Gamma_{f} \cup \Gamma_{l}$, set to

$$
\sigma_{i}=\frac{\pi R^{2}}{N}
$$

where $N$ denotes the total number of atoms on each (111) plane. In calculations, we solve problem (6.1) for $\{\theta\}, \bar{J}_{z}$, and $\lambda$ by means of the NewtonRaphson method, and compute the effective thermal conductivity from the solution as

$$
\bar{\kappa}=\frac{\bar{J}_{z}}{\bar{T}_{z}} .
$$

We recall that nanowires investigated by Li et al. (2003) contain an amorphous surface layer of a thickness of approximately $1 \mathrm{~nm}$ (cf. Fig. 2 of (Li et al., 2003)). We account for this surface layer in our computational model by randomizing the positions of the atoms located near the surface of the nanowire. The thickness of the amorphous layer is set to $1.4 \mathrm{~nm}$ in all simulations. A sample nanowire of radius $R=11 \mathrm{~nm}$ is shown in Fig. 6.1, where atoms in the amorphous surface layer are colored in red.

\subsubsection{Linear kinetics model}

We model the atomic-level thermal conductivity within the linear-kinetics framework presented in Section 3.3. We specifically assume nearest-neighbor 

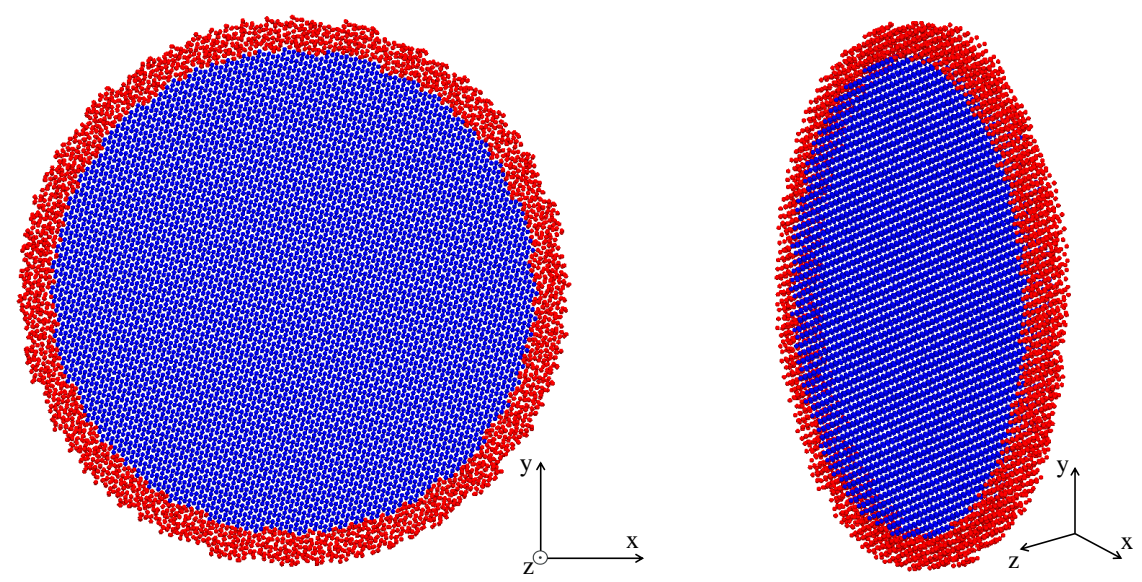

Figure 6.1: Sample Si (111) nanowire of radius $R=11 \mathrm{~nm}$ used in calculations. An amorphous layer of $1.4 \mathrm{~nm}$, generated by randomizing the positions of the atoms, is shown in red.

interactions with transport coefficients

$$
A_{i j}= \begin{cases}A_{\mathrm{amo}}, & \text { if } i \in \Omega_{\mathrm{amo}} \text { or } j \in \Omega_{\mathrm{amo}}, r_{i j}<r_{c} \\ A_{\mathrm{xal}}, & \text { if } i, j \in \Omega \backslash \Omega_{\mathrm{amo}}, r_{i j}<r_{c} \\ 0, & \text { if } r_{i j} \geq r_{c}\end{cases}
$$

where $A_{\text {amo }}$ and $A_{\text {xal }}$ are transport coefficients for the amorphous and crystalline phases, respectively, $\Omega_{\text {amo }}$ is the amorphous boundary layer and $r_{c}$ is a cut-off radius. We set $r_{c}=\left(r_{1}+r_{2}\right) / 2$, where $r_{1}=(\sqrt{3} / 4) a$ and $r_{2}=(\sqrt{2} / 2) a$ are the radii the first and second shells of closest neighbors in the perfect $\mathrm{Si}$ lattice. Evidently, this choice of cut-off radius restricts heat transport to nearest neighbors in the perfect lattice.

\subsubsection{Results and discussion}

We choose the transport coefficients $A_{\text {amo }}$ and $A_{\text {xal }}$ so as to match the dependence of the thermal conductivity on nanowire radius at ambient temperature $T_{0}=320 \mathrm{~K}$ reported by $\mathrm{Li}$ et al. (2003). The transport coefficients thus identified are $A_{\text {amo }}=16.0 \mathrm{nW} / \mathrm{K}$ and $A_{\text {xal }}=0.09 \mathrm{nW} / \mathrm{K}$ and the resulting comparison with experiment is shown in Fig. 6.2. We note that the amorphous transport coefficient $A_{\text {amo }}$ is much smaller than the crystalline transport coefficient $A_{\text {xal }}$. For thin nanowires the amorphous layer has a 


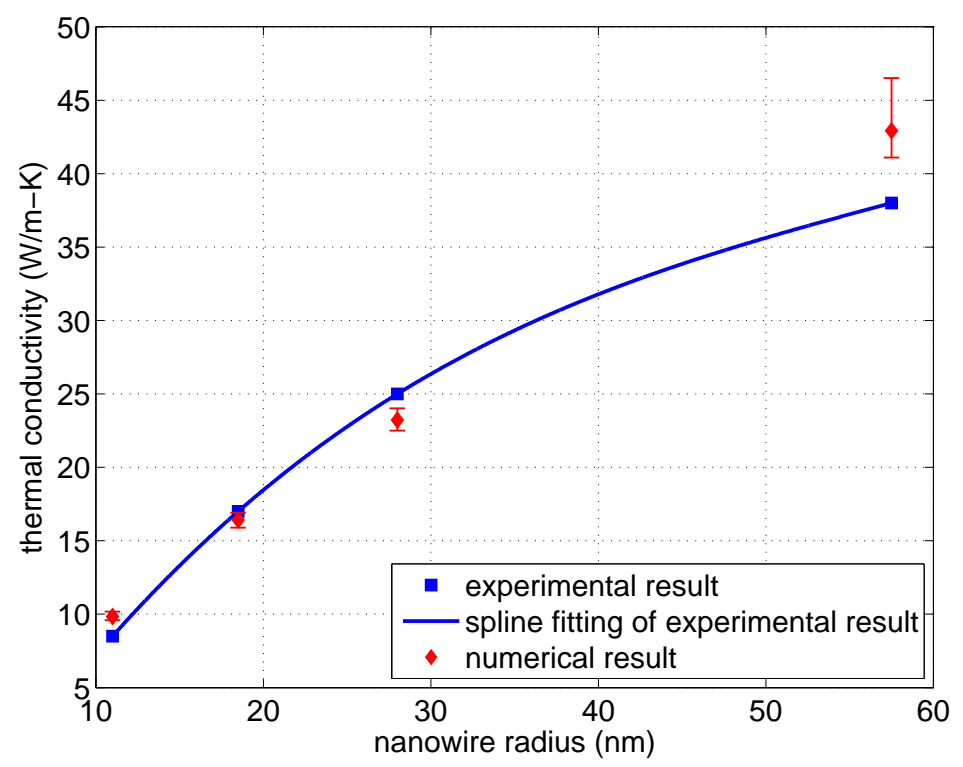

Figure 6.2: Comparison of predicted and measured thermal conductivity of SiNWs of different radii. At each radius, the error bar corresponds to the range of predicted values for 10 random samples. Data from Li et al. (2003).

stronger effect than for for thick nanowires, with the result that the effective thermal conductivity grows with nanowire radius, as observed experimentally. We note that, since the computational model is stochastic due to the randomization of the amorphous layer, the computed thermal conductivities exhibit a certain sample-to-sample scatter clearly evident in the figure. In order to characterize this scatter we repeat the simulations for 10 random samples of the amorphous layer for each radius. The average $\bar{\kappa}$ for each nanowire radius is also calculated and reported in Table 1. Overall, the predicted thermal conductivities are in good agreement with the experimental data, and exemplify the ability of a simple heat transport model to capture atomic-level structure and resulting effective properties.

Fig. 6.3 shows a sample temperature distribution for a nanowire of radius $R=11 \mathrm{~nm}$ at $T_{0}=320 \mathrm{~K}$. As may be seen from the figure, the temperature is nearly uniform in the interior of each nanowire cross-sections and exhibits random fluctuations in the amorphous layer. Simultaneously, the prescribed temperature gradient in the axial direction is clearly visible on the lateral surface of the nanowire. Again, the ability of the model to characterize 


\begin{tabular}{|c|c|c|}
\hline nanowire radius $(\mathrm{nm})$ & $\bar{\kappa}$ - num. (W/m-K) & $\kappa$ - exp. (W/m-K) \\
\hline 11.0 & 9.84 & $8.5 \pm 0.5$ \\
\hline 18.5 & 16.42 & $17.0 \pm 0.5$ \\
\hline 28.0 & 23.22 & $25.0 \pm 0.5$ \\
\hline 57.5 & 42.93 & $38.0 \pm 0.5$ \\
\hline
\end{tabular}

Table 1: Comparison of the average effective conductivity $\bar{\kappa}$ and the experimental measurements of Li et al. (2003) for nanowires of different radii.
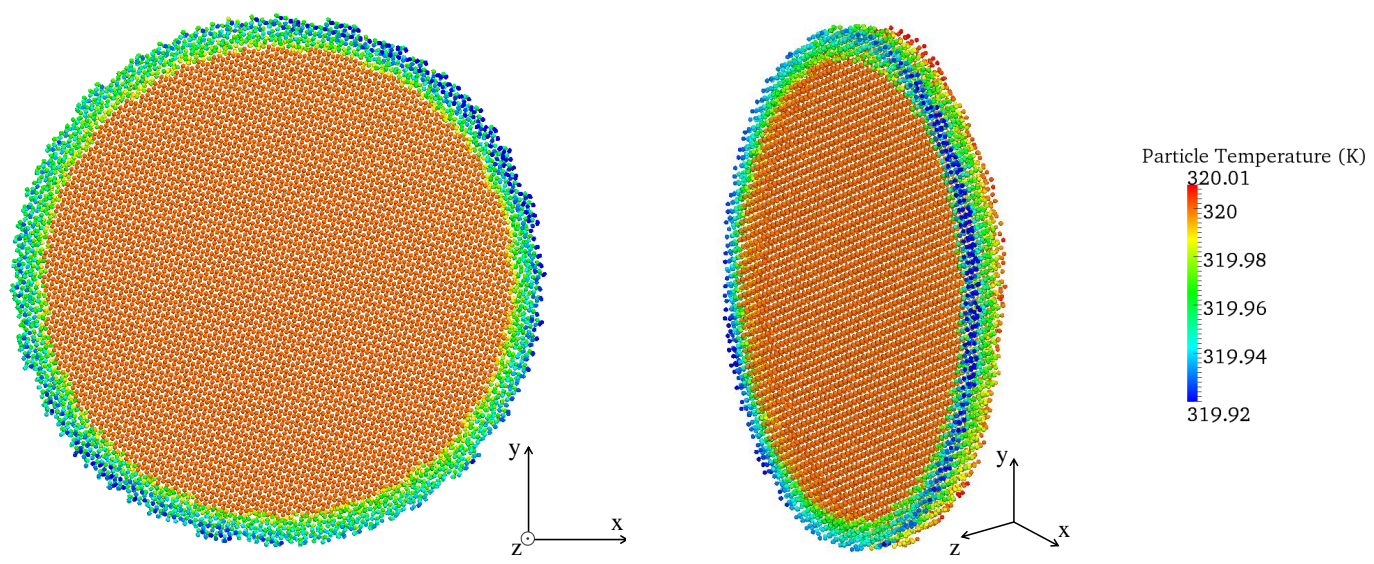

Figure 6.3: Computed temperature distribution for a SiNW of radius $R=11 \mathrm{~nm}$.

atomic-level temperature fields away from equilibrium is noteworthy.

\subsection{Hydrogen diffusion in palladium nanofilms}

Hydrogen is a promising energy carrier as a potential substitute for fossil fuels in future transport applications. In particular, the storage of hydrogen in metals is one of the technologies that has been the focus of extensive research (e. g., Huot (2002); Sakintuna et al. (2007)). In general, hydrogen can be stored in metal hydrides under moderate temperature and pressure at volume densities comparable to, or greater than, liquid hydrogen (see, e. g., Dantzer (2002)). Moreover, the release of hydrogen in metal hydrides is an endothermic process, which makes it inherently safe (Huot, 2002). Among the vast array of metal hydrides known at present, the palladium-hydrogen $(\mathrm{Pd}-\mathrm{H})$ system was the first metal-hydrogen system to be discovered (Graham, 1866). Palladium is capable of absorbing a large atomic percentage 
of hydrogen at room temperature and atmospheric pressure, as required for many applications. The $\mathrm{Pd}-\mathrm{H}$ system exists in two distinct phases: the $\alpha$ phase at low hydrogen concentration (up to $\mathrm{PdH}_{0.015}$ ), and the $\beta$-phase at high hydrogen concentration $\left(\mathrm{PdH}_{0.607}\right.$ and above). Attendant to the $\alpha / \beta$ phase transition, there is a lattice expansion resulting in $10.4 \%$ increase in volume. It is remarkable that the palladium sub-lattice remains face-centered cubic (FCC) in both phases, while the hydrogen atoms occupy interstitial octahedral sites, which themselves define a second FCC sub-lattice. Also in both phases, the hydrogen diffusion coefficient $D_{\mathrm{H}}$ is well described by an Arrhenius law in the temperature range of 230 and $900 \mathrm{~K}$. For bulk palladium at room temperature $(298 \mathrm{~K}), D_{\mathrm{H}}$ is measured to be $3.8 \times 10^{-7} \mathrm{~cm}^{2} / \mathrm{s}$ in $\alpha$-phase and $2.0 \times 10^{-7} \mathrm{~cm}^{2} / \mathrm{s}$ in $\beta$-phase (Adams and Chen, 2011). Recently, extensive research has been devoted to developing and optimizing metal-based nanomaterials for high-speed, high-capacity, reversible hydrogen storage applications (e. g., Huot et al. (1999); Li et al. (2007)). For some nanomaterials, particularly nanofilms, Li and Cheng (1996) and Hagi (1990) showed that these diffusivities may decrease by two to four orders of magnitude.

The absorption and desorption of hydrogen in nanomaterials is characterized by an atomic, deformation-diffusion coupled process on a time scale of the order of seconds to hours - far beyond the time windows accessible to molecular dynamics (MD) and Monte Carlo (MC) methods. Owing to the extensive experimental data presently in existence, hydrogen storage into metal hydrides provides a convenient validation test of the ability of atomic-level empirical mass transport models to account for long transients, such as involved in hydrogen absorption and desorption, while simultaneously affording atomistic realism, including proper accounting of anisotropy, lattice structure, driving forces and phase transitions.

\subsubsection{Computational model}

The aim of this section is to formulate a simple model of absorption and desorption of $\mathrm{H}$ in $\mathrm{Pd}$ which nevertheless demonstrates the ability of the maxent formalism to supply local equilibrium relations and the ability of discrete kinetics to account for long transients. We are thus led to considering a binary system consisting of $\mathrm{Pd}$ atoms occupying a-possibly finite, possibly deformed-perfect FCC lattice whose octahedral interstitial sites are either empty or occupied by $\mathrm{H}$ atoms. For ease of reference, we designate the $\mathrm{Pd}$ atoms in the system by means of an index set $I_{\mathrm{Pd}}$ and the $\mathrm{H}$ atoms by an index 
set $I_{\mathrm{H}}$. For instance, if the system contains $N_{\mathrm{Pd}} \mathrm{Pd}$ atoms and $N_{\mathrm{H}} \mathrm{H}$ atoms, then a enumeration of the atoms gives the index sets $I_{\mathrm{Pd}}=\left\{1, \ldots, N_{\mathrm{Pd}}\right\}$ and $I_{\mathrm{H}}=\left\{1, \ldots, N_{\mathrm{H}}\right\}$. For the system under consideration, the occupancy field $\{\boldsymbol{n}\}$ can be restricted to the octahedral sites, $I_{\mathrm{H}}$ since the Pd sites are always occupied. Hence, $M=1,\{n\}=\left\{n_{i}, i \in I_{\mathrm{H}}\right\}$ and $n_{i}=0$ if the $i$ th octahedral site is empty and $n_{i}=1$ if it is occupied by hydrogen. We again recall that the octahedral sites of the Pd lattice also forms an FCC lattice.

We consider throughout isothermal conditions at fixed temperature $T$, so that $\beta_{i}=\beta=1 / k_{B} T$ uniformly at all atoms of the system. By virtue of this assumption, the energy conservation equation (3.2) and the discrete Fourier's law of heat conduction (3.12a) are rendered superfluous, and the temperature dependence of the response of the system may be regarded as parametric. On the other hand, the discrete Fick's law of diffusion (3.15b) can be restricted to the $\mathrm{H}$ species and thus specialized to

$$
\dot{x}_{i}=-\sum_{j \in I_{\mathrm{H}}, j \neq i} B_{i j} x_{i j} k_{B}\left(\gamma_{i}-\gamma_{j}\right), \quad i \in I_{\mathrm{H}} .
$$

For simplicity, we restrict mass transport to nearest neighbors, whereupon (6.6) simplifies to

$$
\dot{x}_{i}=-B_{0} \sum_{j \in \mathcal{N}_{i}^{(1)}} x_{i j} k_{B}\left(\gamma_{i}-\gamma_{j}\right), \quad i \in I_{\mathrm{H}},
$$

where $\mathcal{N}_{i}^{(k)}$ denotes the $k$-th shell of neighbors of site $i$. Because of lattice symmetry, the bondwise diffusivity coefficient $B_{i j}$ reduces to a lattice-wide constant $B_{0}$, and the discrete-to-continuum relation $(3.20 \mathrm{~b})$ reduces to

$$
D_{\mathrm{H}}=\sum_{j \in \mathcal{N}_{i}^{(1)}} \frac{k_{B}}{6} B_{0}\left(\boldsymbol{r}_{j i} \cdot \boldsymbol{r}_{j i}\right)=k_{B} a_{\mathrm{H}}^{2} B_{0},
$$

where $D_{\mathrm{H}}$ is the isotropic macroscopic diffusivity constant for hydrogen in palladium, which is known experimentally, and $a_{\mathrm{H}}$ is lattice constant of the FCC hydrogen sub-lattice. Inserting (6.8) into (6.7) gives

$$
\dot{x}_{i}=-\frac{D_{\mathrm{H}}}{a_{\mathrm{H}}^{2}} \sum_{j \in \mathcal{N}_{i}^{(1)}} x_{i j}\left(\gamma_{i}-\gamma_{j}\right), \quad i \in I_{\mathrm{H}}
$$


This evolution equation is closed by the equilibrium relation (2.21), which here specializes to

$$
x_{i}=\frac{1}{k_{B}} \frac{\partial \Phi}{\partial \gamma_{i}}(\{\gamma\})
$$

where

$$
\Phi(\{\gamma\})=k_{B} \log \Xi(\{\gamma\})
$$

is the free entropy and

$$
\Xi=\sum_{\{n\} \in \mathcal{O}_{N}} \int_{\Gamma} \mathrm{e}^{-\beta H(\{\boldsymbol{q}\},\{\boldsymbol{p}\},\{n\})+\{\gamma\}^{T}\{n\}} d q d p
$$

is the grand-canonical partition function. In these and subsequent relations, we omit the parametric dependence on temperature for simplicity of notation. In addition, $\mathcal{O}_{N}=\{0,1\}^{N_{\mathrm{H}}}$ since there is one single diffusing species, namely, hydrogen.

We restrict our attention to quasistatic process and, thus, the Hamiltonian $H$ is determined fully by the interatomic potential $V(\{\boldsymbol{q}\},\{n\})$. We specifically assume the empirical potential to be of the Johnson (Johnson, 1989b) form

$$
\begin{aligned}
& V(\{\boldsymbol{q}\},\{n\})=\sum_{i \in I_{\mathrm{Pd}}} F_{\mathrm{Pd}}\left(\rho_{i}\right)+\sum_{i \in I_{\mathrm{H}}} n_{i} F_{\mathrm{H}}\left(\rho_{i}\right)+ \\
& \frac{1}{2} \sum_{i, j \in I_{\mathrm{Pd}}, i \neq j} \Phi_{\mathrm{PdPd}}\left(r_{i j}\right)+\sum_{i \in I_{\mathrm{H}}, j \in I_{\mathrm{Pd}}} n_{i} \Phi_{\mathrm{HPd}}\left(r_{i j}\right)+\frac{1}{2} \sum_{i, j \in I_{\mathrm{H}}, i \neq j} n_{i} n_{j} \Phi_{\mathrm{HH}}\left(r_{i j}\right),
\end{aligned}
$$

with

$$
\rho_{i}=\sum_{j \in I_{\mathrm{Pd}}, j \neq i} f_{\mathrm{Pd}}\left(r_{i j}\right)+\sum_{j \in I_{\mathrm{H}}, j \neq i} n_{j} f_{\mathrm{H}}\left(r_{i j}\right) .
$$

In order to simplify calculations, and in view that the volumetric expansion of the $\mathrm{Pd}-\mathrm{H}$ system during absorbtion is small, we rigidly fix all atoms to their positions $\{\boldsymbol{q}\}_{\text {FCC }}$ in the undeformed FCC pure Pd lattice and its octahedral interstitial $\mathrm{H}$ lattice, whereupon the interatomic potential reduces to

$$
V(\{\boldsymbol{q}\},\{n\}) \approx V\left(\{\boldsymbol{q}\}_{\mathrm{FCC}},\{n\}\right) \equiv U(\{n\}),
$$

i. e., it becomes a function $U(\{n\})$ of the hydrogen occupancy only. By virtue of this rigid approximation, the grand-canonical partition function reduces 
to

$$
\Xi=\sum_{\{n\} \in \mathcal{O}_{N}} \mathrm{e}^{-\beta U(\{n\})+\{\gamma\}^{T}\{n\}} .
$$

In order to simplify calculations further, we expand the embedding energies as

$$
\begin{aligned}
F_{\mathrm{Pd}}(\rho) & \approx F_{\mathrm{Pd}}\left(\rho_{0}^{\mathrm{Pd}}\right)+F_{\mathrm{Pd}}^{\prime}\left(\rho_{0}^{\mathrm{Pd}}\right)\left(\rho-\rho_{0}^{\mathrm{Pd}}\right) \equiv F_{0}^{\mathrm{Pd}}+C_{\mathrm{Pd}}\left(\rho-\rho_{0}^{\mathrm{Pd}}\right), \\
F_{\mathrm{H}}(\rho) & \approx F_{\mathrm{H}}\left(\rho_{0}^{\mathrm{H}}\right)+F_{\mathrm{H}}^{\prime}\left(\rho_{0}^{\mathrm{H}}\right)\left(\rho-\rho_{0}^{\mathrm{H}}\right) \equiv F_{0}^{\mathrm{H}}+C_{\mathrm{H}}\left(\rho-\rho_{0}^{\mathrm{H}}\right)
\end{aligned}
$$

where $\rho_{0}^{\mathrm{Pd}}$ and $\rho_{0}^{\mathrm{H}}$ denote the electron density of $\mathrm{Pd}$ and $\mathrm{H}$ evaluated with the equilibrium $\mathrm{Pd}$ lattice constant. Inserting this approximation into (6.13) and collecting terms we obtain

$$
U(\{n\})=A+\sum_{i \in I_{\mathrm{H}}} B_{i} n_{i}+\sum_{i, j \in I_{\mathrm{H}}, i \neq j} C_{i j} n_{i} n_{j},
$$

where

$$
\begin{aligned}
& A=\sum_{i, j \in I_{\mathrm{Pd}}, i \neq j}\left(C_{\mathrm{Pd}} f_{\mathrm{Pd}}\left(r_{i j}^{\mathrm{FCC}}\right)+\frac{1}{2} \Phi_{\mathrm{PdPd}}\left(r_{i j}^{\mathrm{FCC}}\right)\right)-N_{\mathrm{Pd}}\left(C_{\mathrm{Pd}} \rho_{0}^{\mathrm{Pd}}-F_{0}^{\mathrm{Pd}}\right), \\
& B_{i}=\sum_{j \in I_{\mathrm{Pd}}}\left(C_{\mathrm{Pd}} f_{\mathrm{H}}\left(r_{i j}^{\mathrm{FCC}}\right)+C_{\mathrm{H}} f_{\mathrm{Pd}}\left(r_{i j}^{\mathrm{FCC}}\right)+\Phi_{\mathrm{HPd}}\left(r_{i j}^{\mathrm{FCC}}\right)\right)-C_{\mathrm{H}} \rho_{0}^{\mathrm{H}}+F_{0}^{\mathrm{H}}, \quad i \in I_{\mathrm{H}} \\
& C_{i j}=C_{\mathrm{H}} f_{\mathrm{H}}\left(r_{i j}^{\mathrm{FCC}}\right)+\frac{1}{2} \Phi_{\mathrm{HH}}\left(r_{i j}^{\mathrm{FCC}}\right), \quad i, j \in I_{\mathrm{H}}, i \neq j .
\end{aligned}
$$

Inserting the parametrization and calibration provided in Zhou et al. (2008), for $T=300 \mathrm{~K}, B_{i}$ and $C_{i j}$ evaluate to

$$
B_{i}=-3.2206 \mathrm{eV} \equiv B
$$

and

$$
C_{i j}= \begin{cases}-6.5597 \times 10^{-2} \mathrm{eV} \equiv C_{1}, & \text { if } j \in \mathcal{N}_{i}^{(1)} \\ -1.5826 \times 10^{-2} \mathrm{eV} \equiv C_{2}, & \text { if } j \in \mathcal{N}_{i}^{(2)} \\ -4.4306 \times 10^{-3} \mathrm{eV} \equiv C_{3}, & \text { if } j \in \mathcal{N}_{i}^{(3)} \\ 0, & \text { otherwise }\end{cases}
$$

The constant $A$ drops out from the equilibrium relations and is, therefore, inconsequential. 
Evidently, the simplified energy (6.18) is a quadratic function of the occupancy and, therefore, defines a three-dimensional Ising model. Such models have been extensively studied by a variety of means (cf., e. g., Yeomans (1992)). We recall from those studies that the corresponding grand-canonical partition function (6.16) cannot be computed explicitly in three dimensions. In order to overcome this difficulty, we resort to the variational meanfield theory presented in Section 4. We recall that the trial Hamiltonian in the classical meanfield treatment of the Ising model is linear in the occupancy (cf., e. g., Yeomans (1992)), corresponding to a constant applied field. Likewise, here we consider linear trial Hamiltonians of the form

$$
U_{0}(\{n\})=-k_{B} T \sum_{i \in I_{\mathrm{H}}} \gamma_{0 i} n_{i},
$$

but we allow the mean field $\left\{\gamma_{0}\right\}$ to be non-uniform. A straightforward calculation then gives

$$
\begin{aligned}
& \mathcal{F}\left(\{\gamma\} ;\left\{\gamma_{0}\right\}\right)=\frac{1}{T}\left\langle U-U_{0}\right\rangle_{0}-\Phi_{0}= \\
& \frac{A}{T}+\sum_{i \in I_{\mathrm{H}}}\left(\frac{B_{i}}{T}+k_{B} \gamma_{0 i}\right)\left\langle n_{i}\right\rangle_{0}+ \\
& \sum_{i, j \in I_{\mathrm{H}}, i \neq j} \frac{C_{i j}}{T}\left\langle n_{i}\right\rangle_{0}\left\langle n_{j}\right\rangle_{0}-k_{B} \sum_{i \in I_{\mathrm{H}}} \log \left(\mathrm{e}^{\gamma_{0 i}+\gamma_{i}}+1\right),
\end{aligned}
$$

where

$$
\left\langle n_{i}\right\rangle_{0}=\frac{\mathrm{e}^{\gamma_{0 i}+\gamma_{i}}}{\mathrm{e}^{\gamma_{0 i}+\gamma_{i}}+1}
$$

are the meanfield atomic fractions. Eq. (6.24) can be inverted to give

$$
\gamma_{0 i}=\log \frac{\left\langle n_{i}\right\rangle_{0}}{1-\left\langle n_{i}\right\rangle_{0}}-\gamma_{i}
$$

and the functional (6.23) may be rewritten as

$$
\begin{aligned}
\mathcal{F}\left(\left\{\langle n\rangle_{0}\right\},\{\gamma\}\right) & =\frac{A}{T}+\sum_{i \in I_{H}}\left(\frac{B_{i}}{T}+k_{B} \log \frac{\left\langle n_{i}\right\rangle_{0}}{1-\left\langle n_{i}\right\rangle_{0}}-k_{B} \gamma_{i}\right)\left\langle n_{i}\right\rangle_{0} \\
& +\sum_{i, j \in I_{H}, i \neq j} \frac{C_{i j}}{T}\left\langle n_{i}\right\rangle_{0}\left\langle n_{j}\right\rangle_{0}-k_{B} \sum_{i \in I_{H}} \log \frac{1}{1-\left\langle n_{i}\right\rangle_{0}} .
\end{aligned}
$$


The optimality condition is now

$$
\frac{\partial \mathcal{F}}{\partial\left\langle n_{i}\right\rangle_{0}}=\frac{B_{i}}{T}+k_{B} \log \frac{\left\langle n_{i}\right\rangle_{0}}{1-\left\langle n_{i}\right\rangle_{0}}-k_{B} \gamma_{i}+\sum_{j \in I_{H}, j \neq i} \frac{C_{i j}}{T}\left\langle n_{j}\right\rangle_{0}=0, \quad \forall i \in I_{\mathrm{H}}
$$

which gives the equilibrium relation

$$
\gamma_{i}=\frac{B_{i}}{k_{B} T}+\log \frac{x_{i}}{1-x_{i}}+\sum_{j \in I_{H}, j \neq i} \frac{C_{i j}}{k_{B} T} x_{j}, \quad \forall i \in I_{\mathrm{H}}
$$

where we have used the relation $x_{i}=\left\langle n_{i}\right\rangle_{0}, \forall i \in I_{\mathrm{H}}$.

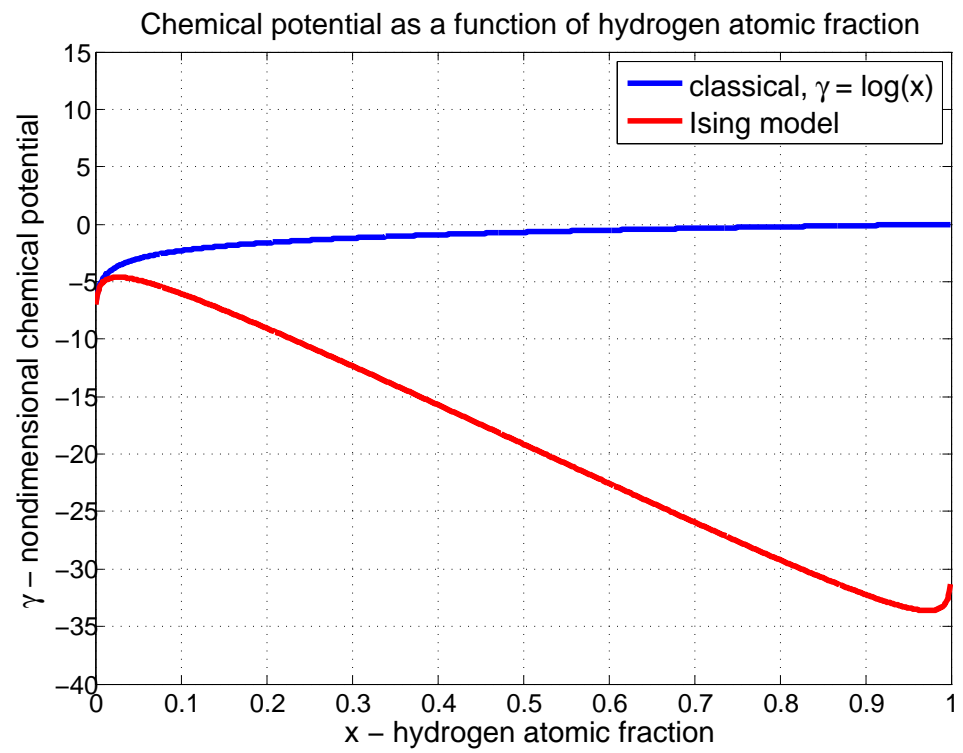

Figure 6.4: Comparison of the equilibrium relation for the Ising model of Pd-H (red) and the classical entropy-of-mixing relation $\gamma=\log x$ (blue).

The equilibrium relation (6.28) is plotted in Fig. 6.4 for a uniform $\mathrm{H}$ atomic fraction and compared with the classical equilibrium relation $\gamma=$ $\log x$ derived from the entropy of mixing. Remarkably, the Ising model results in an up-down-up equilibrium relation characteristic of phase transitions. Specifically, the model predicts the existence of a low concentration phase, which may be identified with the $\alpha$ phase, and a high concentration phase, which may likewise be identified with the $\beta$ phase. The ability of the simple 
Ising model to capture the phase structure of $\mathrm{Pd}-\mathrm{H}$ is remarkable. However, in the simulations that follow the hydrogen concentrations remain relatively low and the system remains in the $\alpha$ phase throughout. Simulations taking the system into the $\beta$-phase, which should also engender fine $\alpha$ - $\beta$ phase mixtures, would be of considerable interest but are beyond the scope of the present paper.

\subsubsection{Model validation for hydrogen desorption in Pd(111) thin films}

We proceed to compare the predictions of the model just described with the experimental study of $\mathrm{Li}$ and Cheng (1996) on hydrogen diffusion in $\mathrm{Pd}$ thin films. The specimens tested by Li and Cheng (1996) were three Pd (111) films fabricated by physical vapor deposition of $\mathrm{Pd}$ onto a gold-coated nickel substrate. The thicknesses of the films were $220 \AA, 460 \AA$, and $1350 \AA$. For each specimen, the hydrogen diffusion coefficient $D_{\mathrm{H}}$ for $\alpha$-phase $\mathrm{Pd}-\mathrm{H}$ was determined by an electrochemical stripping method. Specifically, the time history of electric current, which is proportional to the spatial gradient of hydrogen atomic fraction, was measured at film surface during desorption, and $D_{\mathrm{H}}$ was derived by an appeal to Fick's law of diffusion. The full time histories of electric current for the $460 \AA$ and $1350 \AA$ films during hydrogen desorption were reported in $\mathrm{Li}$ and Cheng (1996), and these time histories provide the main basis for the present validation.

The surface area of the films was $1.2 \mathrm{~cm}^{2}$ and, therefore, the hydrogen desorption process was essentially one-dimensional in the thickness direction. In view of this geometry, in calculations we take the hydrogen atomic fractions $\{x\}$ and chemical potentials $\{\gamma\}$ to be constant on (111) planes. We then denote $x_{k}$ and $\gamma_{k}, k=1, \ldots, N$, the atomic fraction and chemical potential on the $k$-th (111) plane, where $N$ is the number of (111) planes in the sample. The particular plane $k=1$ corresponds to the film surface, in contact with the electrolyte solution or hydrogen sink, and $k=N$ corresponds to the opposite side of the film in contact with the substrate. Taking into account the FCC lattice geometry, the discrete kinetic equation (6.9) and the meanfield optimality condition (6.28) reduce to

$$
\dot{x}_{k}=\frac{3 D_{H}}{a_{\mathrm{H}}^{2}}\left(\frac{x_{k}+x_{k-1}}{2}\left(\gamma_{k-1}-\gamma_{k}\right)+\frac{x_{k}+x_{k+1}}{2}\left(\gamma_{k+1}-\gamma_{k}\right)\right) \text {, }
$$


for $k=2, \ldots, N-1$, and

$$
\begin{aligned}
\gamma_{k} & =\log \frac{1-x_{k}}{x_{k}}-\frac{B}{k_{B} T}-\frac{1}{k_{B} T}\left[\left(6 C_{1}+6 C_{3}\right) x_{k}\right. \\
& \left.+\left(3 C_{1}+3 C_{2}+6 C_{3}\right)\left(x_{k-1}+x_{k+1}\right)+3 C_{3}\left(x_{k-2}+x_{k+2}\right)\right]
\end{aligned}
$$

for $k=1, \ldots, N$, where $B$ and $C_{l}, l=1,2,3$ are defined in (6.20) and (6.21), respectively. Eqs. (6.29) and (6.30) are closed by initial and boundary conditions proposed by Li and Cheng (1996) as representative of their experiment, namely,

$$
\begin{aligned}
& x_{k}(0)=x_{0}, \quad k=1, \ldots, N ; \\
& x_{1}(t)=x^{*}, \quad 0<t<t_{\max } ; \\
& x_{N-1}(t)=x_{N}(t), \quad 0<t<t_{\max } .
\end{aligned}
$$

We specifically simulate the films of thickness $L=460 \AA$ and $1350 \AA$ for which time-dependent data is provided by Li and Cheng (1996). In both cases, $x_{0}$ is the saturation atomic fraction of hydrogen at a cathodically polarized potential of $-0.830 \mathrm{~V}$ at which the films are held before desorption starts. The measurements of Hagi (1990) (see Fig. 1 therein) give $x_{0}=0.009$. Furthermore, $x^{*}$ denotes the atomic fraction of hydrogen at the surface of the film. The value of $x^{*}$ is not direct provided by Li and Cheng (1996). However, an estimate for $x^{*}$ and $D_{H}$ can be readily derived from Figs. 6(a) and 7(a) therein by an appeal to Fick's second law. The estimated value of $x^{*}$ used in calculations, together with the remaining parameters of the model, are collected in Table 2

\begin{tabular}{|l|c|c|c|c|}
\hline & $L(\AA)$ & $N$ & $x^{*}$ & $D_{\mathrm{H}}\left(\AA^{2} / \mathrm{s}\right)$ \\
\hline Case I & 460 & 206 & 0.0019 & $2.1 \times 10^{5}$ \\
\hline Case II & 1350 & 603 & 0.0047 & $1.56 \times 10^{6}$ \\
\hline
\end{tabular}

Table 2: Coefficients used in the hydrogen desorption simulations

All experiments were performed at $T=300 \mathrm{~K}$. We additionally set the lattice parameter $a_{\mathrm{H}}$ to $3.885 \AA$ based on EAM potential predictions for a perfect FCC Pd lattice at that temperature (Zhou et al., 2008). Eq. (6.29) is discretized in time by a simple Forward Euler scheme. The computational time step is set to $\Delta t=5.0 \times 10^{-6} \mathrm{~s}$ and $1.0 \times 10^{-6} \mathrm{~s}$ in Case I and II, respectively. In both cases, the simulations are terminated at $t_{\max }=1.0 \mathrm{~s}$. 


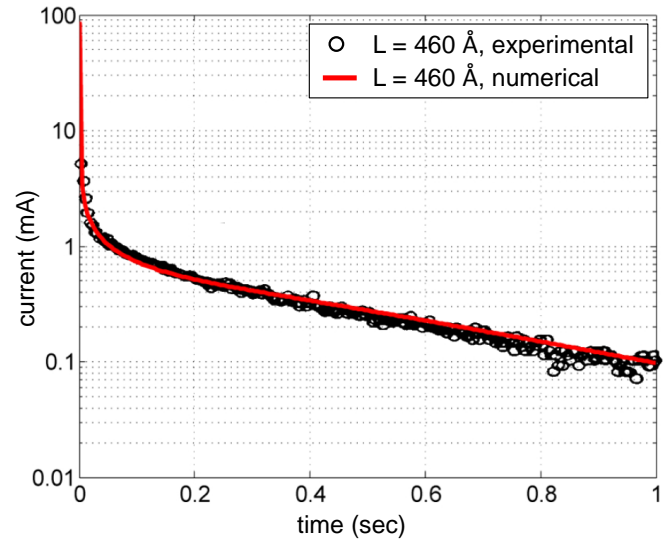

(a)

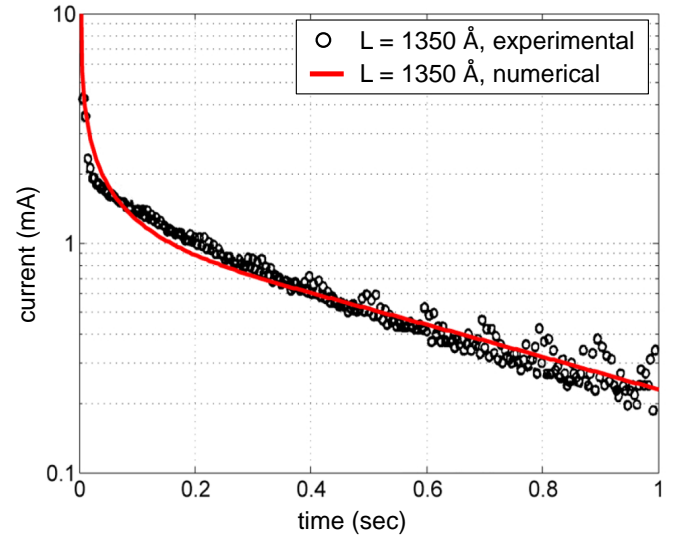

(b)

Figure 6.5: Hydrogen desorption from palladium. Comparison between predicted and experimental electric-current time histories at film surface. a) $460 \AA \mathrm{Pd}$ film. b) 1350 $\AA$ Pd film.

In order to compare with the experimental measurements, the hydrogen atomic fractions at film surface are converted into electric current using the relation (Boes and Züchner, 1976)

$$
I(t)=\frac{4 F S D_{\mathrm{H}}}{N_{A} a_{H}^{3}}\left(\frac{x_{2}(t)-x_{1}(t)}{h_{(111)}}\right),
$$

where $I(t)$ denotes electric current at film surface, $N_{A}$ denotes the Avogadro constant, $F$ denotes the Faraday constant, $S=1.2 \mathrm{~cm}^{2}$ is surface area, and $h_{(111)}=(\sqrt{3} / 3) a_{\mathrm{H}}$ is the lattice spacing of (111) planes. A comparison of $I(t)$ predicted by the simulations and the corresponding experimental data is shown in Fig. 6.5 for $0<t<t_{\max }$. In both cases, the predicted current is in good agreement with the experimental measurements, both as regards the initial transient as well as the long-term behavior. For the thicker film, the predicted initial transient is somewhat slower than observed experimentally. These limitations notwithstanding, the present example demonstrates the ability of the theory to characterize slow transport processes over exceedingly long times based on input from the atomic scales, including lattice geometry, interatomic potentials and transport constants. 


\section{Summary and concluding remarks}

We have formulated of a theory of non-equilibrium statistical thermodynamics for ensembles of atoms or molecules that address that theoretical and computational gap. The theory is an application of Jayne's maximum entropy principle (Jaynes, 1957a,b), which allows the statistical treatment of systems away from equilibrium. In particular, neither temperature nor atomic fractions are required to be uniform but instead are allowed to take different values from particle to particle. In addition, we mirror the classical Coleman-Noll method of continuum thermodynamics (Coleman and Noll, 1963) and derive a dissipation inequality expressed in terms of discrete thermodynamic fluxes and forces. This discrete dissipation inequality effectively sets the structure for discrete kinetic potentials that couple the microscopic field rates to the corresponding driving forces, thus resulting in a closed set of equations governing the evolution of the system. We complement the general theory with a variational meanfield theory that provides a basis for the formulation of computationally tractable approximations. We have presented several validation cases that demonstrate the range and scope of the method and assess its fidelity and predictiveness. These validation cases are characterized by the need or desirability to account for atomic-level properties, including lattice structure, interatomic potentials, mixing and phase stability, among others, while simultaneously entailing time scales much longer than those accessible to direct molecular dynamics. The ability of simple meanfield models to reproduce equilibrium properties of alloys, size and temperature effects in heat conduction on silicon nanowires and phase-transitions and long-term behavior in hydrogen desorption from palladium thin films, is remarkable.

Several trade-offs pertaining to the formulation of the theory bear further discussion. Thus, one of the main advantages of the theory is that it bypasses the need to track thermal vibrations and individual particle hops explicitly, thereby greatly extending the time scales amenable to study. The counterpoint to this advantage is that local transport properties are no longer predicted but must be modeled empirically and calibrated experimentally. However, the accurate prediction of transport properties from first principles is exceedingly challenging and, thus, the ability to integrate measured kinetic properties into calculations, including thermal conductivities and diffusivities, may indeed be construed as an advantage. This trade-off is similar to the interplay between first-principles quantum mechanical approaches in 
condensed-matter physics, an accurate but often intractable theory of material structure, and empirical interatomic potentials such as the EmbeddedAtom Method, which are subject to modeling and computationally efficient. Likewise, whereas there are general theories that supply an understanding of transport phenomena, there is presently a paucity of empirical atomiclevel transport models similar in spirit and scope to empirical interatomic potentials, and further development of such models is much to be desired.

As already mentioned, the narrow aim of this paper focuses on the formulation, demonstration and validation of the theory. To this end, we have formulated simple meanfield and kinetic models that nevertheless suffice to demonstrate the range and scope of the theory and provide a measure of validation thereof. Some elements of the theory, particularly meanfield approximation theory, are intended to supply a basis for a systematic improvements of the models and for a general numerical implementation. Thus, a general scheme for systematically formulating increasingly accurate meanfield approximations relies on cluster expansions (cf., e. g., Yeomans (1992)). In this manner, meanfield models become controlled approximation schemes resulting in convergent sequences of approximations. Likewise, the variational structure of the theory provides a basis for variational time discretization cf., e. g., Ortiz and Stainier (1999); Yang et al. (2006)) and for spatial coarsegraining, e. g., by recourse to the quasi-continuum method (Tadmor et al., 1996; Knap and Ortiz, 2001; Kulkarni et al., 2008; Ariza et al., 2012). These and other extensions suggest worthwhile avenues for further work.

\section{Acknowledgements}

G.V. and M.O. gratefully acknowledge the support of the Department of Energy (DoE) National Nuclear Security Administration (NNSA) under Award Number DE-FC52-08NA28613 through Caltech's ASC/PSAAP Center for the Predictive Modeling and Simulation of High Energy Density Dynamic Response of Materials as well as the support from the Lawrence Livermore National Laboratory (LLNL) through Sponsor Award No. B579041 from Prime Contract No. DE-AC52-07NA27344. M.O. and K.W. gratefully acknowledge support from the U. S. Army Research Laboratory (ARL) through the Materials in Extreme Dynamic Environments (MEDE) Collaborative Research Alliance (CRA) under Award Number W911NF-11-R-0001.

Support from the Caja Madrid foundation for the sabbatical stay of I.R. at 
Caltech is gratefully acknowledged. P.A. gratefully acknowledges the support of the Ministerio de Economía y Competitividad of Spain (DPI2012-32508).

\section{Appendix A. Derivation of the long-wavelength limit of the dis- crete kinetic equations}

For systems characterized by short-ranged linear transport coefficients $A_{i j}$ and $B_{i j}^{l}$, we expect the long-wavelength limit of the transport equations (3.18) to be of the form

$$
\begin{aligned}
& T \dot{\eta}=-\rho c_{v} \boldsymbol{v} \cdot \nabla T+\nabla \cdot(\boldsymbol{\kappa} \nabla T), \\
& \dot{c}_{l}=-\frac{\boldsymbol{v}_{l}}{R T} \cdot \nabla \mu_{l}+\nabla \cdot\left(\frac{\boldsymbol{D}_{l} c_{l}}{R T} \nabla \mu_{l}\right)
\end{aligned}
$$

where $T$ is the continuum absolute temperature, $\eta$ is the entropy density per unit volume, $\rho$ is the mass density, $c_{v}$ is the heat capacity per unit mass at constant volume, $\boldsymbol{v}$ is an advection velocity for heat, $\boldsymbol{\kappa}$ is the-possibly anisotropic - thermal-conductivity tensor, $c^{l}$ is the continuum concentration of species $l, \boldsymbol{v}_{l}$ is a drift velocity for species $l, R$ universal gas constant and $\boldsymbol{D}^{l}$ is the - possibly anisotropic-diffusivity tensor of species $l$. We identify in (A.1a) the heat advection-diffusion equation and in (A.1b) the mass advection-diffusion equation.

We start by considering the heat transfer process. Suppose that the continuum fields are slowly varying on the atomistic scale. We define corresponding discrete entropy and temperature fields as

$$
\begin{aligned}
& s_{i}(t)=\eta\left(\boldsymbol{q}_{i}, t\right) \Omega_{i}, \\
& \theta_{i}(t)=T\left(\boldsymbol{q}_{i}, t\right)
\end{aligned}
$$

where $\Omega_{i}$ is an atomic volume for particle $i$. Suppose that the transport coefficients $A_{i j}$ are short ranged. Then, expanding to leading order we have

$$
\begin{aligned}
& \theta_{j} \sim \theta_{i}+\nabla T_{i} \cdot \boldsymbol{r}_{j i}+\text { h. o. t., } \\
& \beta_{j} \sim \beta_{i}-\frac{\nabla T_{i}}{k_{B} T_{i}^{2}} \cdot \boldsymbol{r}_{j i}+\left(\frac{\nabla T_{i} \otimes \nabla T_{i}}{k_{B} T_{i}^{3}}-\frac{1}{2} \frac{\nabla \nabla T_{i}}{k_{B} T_{i}^{2}}\right) \cdot\left(\boldsymbol{r}_{j i} \otimes \boldsymbol{r}_{j i}\right)+\text { h. o. t., }
\end{aligned}
$$

where we write $T_{i}(t) \equiv T\left(\boldsymbol{q}_{i}, t\right), \nabla T_{i}(t) \equiv \nabla T\left(\boldsymbol{q}_{i}, t\right)$ and $\nabla \nabla T_{i}(t) \equiv \nabla \nabla T\left(\boldsymbol{q}_{i}, t\right)$ for short, and we denote by $\boldsymbol{r}_{j i}$ the relative position vector between particles 
$i$ and $j$. Inserting (A.3) into (3.18a) we have, to leading order,

$$
T_{i} \dot{\eta}_{i} \Omega_{i} \sim\left(\sum_{\langle i, j\rangle} A_{i j} \boldsymbol{r}_{j i}\right) \cdot \nabla T_{i}+\left(\sum_{\langle i, j\rangle} \frac{1}{2} A_{i j} \boldsymbol{r}_{j i} \otimes \boldsymbol{r}_{j i}\right) \cdot \nabla \nabla T_{i}
$$

Comparison of this equation with (A.1a) finally results in the relations (3.19).

Consider now the mass transfer process. We again define corresponding discrete atomic fraction and chemical-potential fields as

$$
\begin{aligned}
x_{i}^{l}(t) & =c_{l}\left(\boldsymbol{q}_{i}, t\right) \Omega_{i}, \\
\gamma_{i}^{l}(t) & =\frac{\mu_{l}\left(\boldsymbol{q}_{i}, t\right)}{R T\left(\boldsymbol{q}_{i}, t\right)}
\end{aligned}
$$

where $\Omega_{i}$ is an atomic volume for particle $i$. Suppose, as before, that the transport coefficients $B_{i j}^{l}$ are short ranged. Then, expanding to leading order we have

$$
\begin{aligned}
& x_{j}^{l} \sim x_{i}^{l}+\Omega_{i} \nabla c_{i}^{l} \cdot \boldsymbol{r}_{j i}+\text { h. o. t., } \\
& \gamma_{j}^{l} \sim \gamma_{i}^{l}+\frac{\nabla \mu_{i}^{l}}{R T_{i}} \cdot \boldsymbol{r}_{j i}+\frac{1}{2} \frac{\nabla \nabla \mu_{i}^{l}}{R T_{i}} \cdot\left(\boldsymbol{r}_{j i} \otimes \boldsymbol{r}_{j i}\right)+\text { h. o. t. },
\end{aligned}
$$

where we write $\nabla \mu_{i}^{l}(t) \equiv \nabla \mu_{l}\left(\boldsymbol{q}_{i}, t\right), \nabla \nabla \mu_{i}^{l}(t) \equiv \nabla \nabla \mu_{l}\left(\boldsymbol{q}_{i}, t\right), \nabla c_{i}^{l}(t) \equiv$ $\nabla c_{l}\left(\boldsymbol{q}_{i}, t\right)$ and $T_{i}(t) \equiv T\left(\boldsymbol{q}_{i}, t\right)$ for short. Inserting (A.6b) and (A.6a) into (3.18b) and dividing through by $\Omega_{i}$ we obtain, to leading order,

$$
\begin{aligned}
\dot{c}_{i}^{l} & \sim\left(\sum_{\langle i, j\rangle} B_{i j}^{l} k_{B} \boldsymbol{r}_{j i}\right) \cdot\left(c_{i}^{l} \frac{\nabla \mu_{i}^{l}}{R T_{i}}\right) \\
& +\left(\sum_{\langle i, j\rangle} \frac{k_{B}}{2} B_{i j}^{l}\left(\boldsymbol{r}_{j i} \otimes \boldsymbol{r}_{j i}\right)\right) \cdot\left(c_{i}^{l} \frac{\nabla \nabla \mu_{i}^{l}}{R T_{i}}+\nabla c_{i}^{l} \otimes \frac{\nabla \mu_{i}^{l}}{R T_{i}}\right) .
\end{aligned}
$$

Comparison of this equation with (A.1b) results in the relations (3.20).

\section{References}

Ackland, G., Vitek, V., 1990. Many-Body Potentials and Atomic Scale Relaxations in Noble-Metal Alloys. Phys. Rev. B 41, 10324-10333. 
Adams, B.D., Chen, A., 2011. The role of palladium in a hydrogen economy. Materials Today 14, 282-289.

Ariza, M.P., Romero, I., Ponga, M., Ortiz, M., 2012. HotQC simulation of nanovoid growth under tension in copper. International Journal of Fracture $174,75-85$.

Askar, A., 1986. Lattice dynamical foundations of continuum theories : elasticity, piezoelectricity, viscoelasticity, plasticity. Series in theoretical and applied mechanics, World Scientific, Singapore, Philadelphia.

Bidwell, L., 1964. Unit-Cell Dimensions of Ni-Pd alloys at 25 and 900 C. Acta Crystallogr. 17, 1473-1474.

Boes, N., Züchner, H., 1976. Electrochemical methods for studying diffusion, permeation and solubility of hydrogen in metals. J. Less Common Met. 49, $223-240$.

Bozzolo, G., R., N., Abel, P.E., 2007. Applied Computational Material Modeling. Theory, Simulation and Experiments. Springer.

Callen, H., 1985. Thermodynamics and an Introduction to Thermostatistics. John Wiley \& Sons.

Casimir, H.B.G., 1945. On onsager principle of microscopic reversibility. Reviews of Modern Physics 17, 343-350.

Chen, R., Hochbaum, A.I., Murphy, P., Moore, J., Yang, P., Majumdar, A., 2008. Thermal conductance of thin silicon nanowires. Physical Review Letters 101.

Coleman, B.D., Noll, W., 1963. The thermodynamics of elastic materials with heat conduction and viscosity. Archive for Rational Mechanics and Analysis 13, $167-178$.

Cui, Y., Lauhon, L., Gudiksen, M., Wang, J., Lieber, C., 2001. Diameter-controlled synthesis of single-crystal silicon nanowires. Applied Physics Letters 78, 22142216.

Dantzer, P., 2002. Properties of intermetallic compounds suitable for hydrogen storage applications. Mater. Sci. and Eng. A 329, 313-320.

De Groot, S.R., Mazur, P., 1962. Non-equilibrium thermodynamics. NorthHolland, Amsterdam. 
Derry, G., Wan, R., 2004. Comparison of Surface Structure and Segregation in AgAu and NiPd Alloys. Surf. Sci. 566-568, 862-868.

Doerk, G.S., Carraro, C., Maboudian, R., 2010. Single Nanowire Thermal Conductivity Measurements by Raman Thermography. ACS NANO 4, 4908-4914.

Donadio, D., Galli, G., 2009. Atomistic Simulations of Heat Transport in Silicon Nanowires. Physical Review Letters 102.

Foiles, S., M., B., Daw, M., 1986. Embedded Atom Method Functions for the FCC Metals Cu, Ag, Au, Ni, Pd, Pt an their Alloys. Phys. Rev. B 33, 7983-7991.

Gill, S., 2010. Nonequilibrium Molecular Dynamics and Multiscale Modeling of Heat Conduction in Solids (Trends in Computational Nanomechanics, v. 9). Springer.

Girifalco, L., 2000. Statistical Mechanics of Solids. Oxford University Press.

Graham, T., 1866. On the absorption and dialytic separation of gases by colloid septa. Phil. Trans. Royal Soc. (London) 156, 399-439.

Hagi, H., 1990. Diffusion coefficient of hydrogen in palladium films prepared by RF sputtering. Mater. Trans. JIM 31, 954-958.

Helms, C., Yu, K., 1975. Determination of the Surface Composition of the CuNi alloys for Clean and Adsorbate-Covered Surfaces. J. Vac. Sci. Technol. 12, $276-278$.

Hicks, L., Dresselhaus, M., 1993. Thermoelectric figure of merit of a onedimensional conductor. Physical Review B 47, 16631-16634.

Hill, T., 1962. Introduction to Statistical Thermodynamics. Addison Wesley.

Hill, T., 1987. Statistical Mechanics: Principles and Selected Applications. Dover Publications.

Hochbaum, A.I., Chen, R., Delgado, R.D., Liang, W., Garnett, E.C., Najarian, M., Majumdar, A., Yang, P., 2008. Enhanced thermoelectric performance of rough silicon nanowires. Nature 451, 163-U5.

Huot, J., 2002. Hydrogen in metals. New Trends in Intercalation Compounds for Energy Storage 61, 109-143. 
Huot, J., Liang, G., Boily, S., Van Neste, A., Schulz, R., 1999. Structural study and hydrogen sorption kinetics of ball-milled magnesium hydride. J. Alloys and Compounds 293, 495-500.

Jaynes, E.T., 1957a. Information theory and statistical mechanics i. Phys. Rev. II 106, 620-630.

Jaynes, E.T., 1957b. Information theory and statistical mechanics ii. Phys. Rev. II 108, 171-190.

Johnson, R., 1988. Analytic Nearest Neighbor Model for fcc Metals. Phys. Rev. B 37, 3924-3931.

Johnson, R., 1989a. Alloy Models with the Embedded Atom Method. Phys. Rev. B 39, 12554-12559.

Johnson, R.A., 1989b. Alloy models with the embedded-atom method. Phys. Rev. B 39, 12554-12559.

King, T., Donnelly, R., 1985. Surface Compositions and Composition Profiles of AgAu (100), (110) and (111) Surfaces Determined Quantitatively by Auger Electron Spectroscopy. Surf. Sci. 151, 374-399.

Knap, J., Ortiz, M., 2001. An analysis of the quasicontinuum method. J. Mech. Phys. Solids 49, 1899.

Kulkarni, Y., Knap, J., Ortiz, M., 2008. A Variational Approach to Coarse Graining of Equilibrium and Non-Equilibrium Atomistic Description at Finite Temperature. J. Mech. Phys. Solids 56, 1417-1449.

Li, D., Wu, Y., Kim, P., Shi, L., Yang, P., Majumdar, A., 2003. Thermal conductivity of individual silicon nanowires. Appl. Phys. Lett. 83, 2934-2936.

Li, W., Li, C., Ma, H., Chen, J., 2007. Magnesium nanowires: enhanced kinetics for hydrogen absorption and desorption. J. Am. Chem. Soc. 129, 6710-6711.

Li, Y., Cheng, Y.T., 1996. Hydrogen diffusion and solubility in palladium thin films. Int. J. Hydrogen Energy 21, 281-291.

Malvern, L.E., 1969. Introduction to the Mechanics of a Continuum Medium. Prentice-Hall, Englewood Cliffs, N.J.

Markussen, T., Jauho, A.P., Brandbyge, M., 2008. Heat conductance is strongly anisotropic for pristine silicon nanowires. Nano Letters 8, 3771-3775. 
Marquis, E., Hyde, J., Saxey, D., Lozano-Perez, S., de Castro, V., Hudson, D., Williams, C., Humphry-Baker, S., Smith, G., 2009. Nuclear Reactor Materials at the Atomic Scale. Materials Today 12, 30-37.

Onsager, L., 1931a. Reciprocal relations in irreversible processes. i. Physical Review 37, 405-426.

Onsager, L., 1931b. Reciprocal relations in irreversible processes. ii. Physical Review 38, 2265-2279.

Ortiz, M., Stainier, L., 1999. The variational formulation of viscoplastic constitutive updates. Computer Methods in Applied Mechanics and Engineering 171, 419-444.

Ponga, M., Romero, I., Ortiz, M., Ariza, M., 2012. Finite temperature nanovoids evolution in fcc metals using quasicontinuum method. Key Engineering Materials $488-489,387-390$.

Rockafellar, R.T., 1970. Convex analysis. volume 28. Princeton University Press, Princeton, N.J.

Sakintuna, B., Lamari-Darkrim, F., Hirscher, M., 2007. Metal hydride materials for solid hydrogen storage: a review. Int. J. Hydrogen Energy 32, 1121-1140.

Sakurai, T., Hashizume, T., A., J., Sakai, A., 1985. New Results in Surface Segregation of NiCu Binary Alloys. Phys. Rev. Lett. 55, 514-517.

Shi, L., Li, D., Yu, C., Jang, W., Kim, D., Yao, Z., Kim, P., Majumdar, A., 2003. Measuring thermal and thermoelectric properties of one-dimensional nanostructures using a microfabricated device. J. Heat Transfer 125, 881-888.

Simmons, G., 1971. Single Crystal Elastic Constants and Calculated Aggregate Properties: A Handbook. The MIT Press.

Simmons, R., Balluffi, R., 1963. Measurement of Equilibrium Concentration of Vacancies in Copper. Phys. Rev. 129, 1533.

Stroud, A.H., 1971. Approximate Calculation of Multiple Integrals. Prentice-Hall.

Sutton, A., Mulheran, P., Stoneham, A., 1992. Direct Free Energy Minimization Methods: Application to Grain Boundaries. Philosophical Transactions: Physical Sciences and Engineering 341, 233-245. 
Tadmor, E., Ortiz, M., Phillips, R., 1996. Quasicontinuum analysis of defects in solids. Philos. Mag. A 73, 1529.

Toloukian, R., 1975. Physical Properties of Matter. Thermal Expansion - Metallic Elements \& Alloys - vol 12. The TPRC Data Series.

Venturini, G., 2011. Topics in multiscale modeling of metals and metallic alloys. Ph.D. thesis. California Institute of Technology. http://resolver.caltech.edu/CaltechTHESIS:11222010-114324484.

Voter, A., Montalenti, F., Germann, T., 2002. Extending the Time Scale in Atomistic Simulation of Materials. Annu. Rev. Mater. Res. 32, 321-346.

Wandelt, K., Brundle, C., 1981. Evidence for Crystal-Fsce Specificity in Surface Segregation of CuNi Alloys. Phys. Rev. Lett. 46, 1529-1532.

Wang, H., Najafabadi, R., Srolovitz, D., 1992. (100) Surface Segregation in Cu-Ni Alloys. Phys. Rev. B 45, 12028-12042.

Weiner, J., 2002. Statistical Mechanics of Elasticity. Dover Publications.

Yang, Q., Stainier, L., Ortiz, M., 2006. A Variational Formulation of the Coupled Thermo-Mechanical Boundary-Value Problem for General Dissipative Solids. J. Mech. Phys. Solids 54, 401-424.

Yeomans, J.M., 1992. Statistical Mechanics of Phase Transitions. Oxford University Press.

Zhou, X.W., Zimmerman, J.A., Wong, B.M., Hoyt, J.J., 2008. An embedded-atom method interatomic potential for Pd-H alloys. J. Mater. Res. 23, 707-718.

Ziman, J.M., 1960. Electrons and phonos. The international series of monographs on physics, Oxford University Press.

Zubarev, D.N., 1974. Non-equilibrium statistical thermodynamics. Consultants Bureau. 